\title{
Isoradial meshes: covering elastic gridshells with planar facets
}

\author{
Cyril Douthe $^{\mathrm{a}, *}$, Romain Mesnil ${ }^{\mathrm{a}}$, Hugo Orts ${ }^{\mathrm{a}}$, Olivier Baverel ${ }^{\mathrm{a}}$ \\ ${ }^{a}$ Université Paris-Est, Laboratoire Navier, École des Ponts ParisTech, IFSTTAR, \\ CNRS, Champs-sur-Marne, France
}

\begin{abstract}
Elastic gridshells are structures made of flat two-way grids which are deformed elastically before they are braced and which afterwards mechanically behave like continuous shells. Gridshells present some advantages in terms of manufacturing, lightness and time of assembly. Their covering remains however a technical issue. The present article proposes hence an alternative method to cover them by planar quadrilateral facets, which could also be used as natural bracing if connected properly. It relies on the duality between a certain family of circular meshes with a unique radius and some Tchebycheff nets. The approach is versatile and allows for the design of a large variety of shapes from two curves in space. Real time numerical tools are developed for open and closed curves as well as a strategy for umbilical points. The relaxation of the Tchebycheff net shows finally that an equilibrium configuration can be found in the vicinity of the planar quadrilateral mesh (PQ-Mesh) which confirm the practical feasibility of elastic gridshells covered with planar facets.
\end{abstract}

\footnotetext{
${ }^{*}$ Corresponding author

Email address: cyril.douthe@enpc.fr (Cyril Douthe)
} 
Keywords: Structural morphogenesis, architectural geometry, Tchebycheff net, circular mesh, PQ-mesh, umbilic, dynamic relaxation.

\section{Structural morphogenesis of elastic gridshells}

One of architecture's latest paradigms is the design of complexly-shaped structures based on a large formal freedom, usually called free-form structures. However the physical constraints in terms of constructability and mechanical performances still are a limitation to such innovative creation [1]. To tackle this issue, two fundamentally opposite philosophies emerged:

- the top-down approach which consists in some post-rationalisation process of a grid or a mesh mapped on a pre-defined surface through different optimisation methods;

- the bottom-up approach which consists in direct construction processes of the discrete objects (grid or mesh) without preliminary knowledge of the surface through purely geometrical methods or mixing geometry and physics in so-called form-finding methods.

In the latter case, the surface and the mesh are generated simultaneously which, to our opinion, gives more insight to the designer on the structure and envelop that he is sketching. There is a lack for such methods for elastic gridshells and this paper intends at remedying this shortfall.

The specificity of elastic gridshells relies in their construction process. Indeed they are made of flat two-way grids which are deformed elastically before they are braced and which afterwards mechanically behave like continuous shells $[2,3]$. This deformability of the grid is insured by:

- the absence of shear rigidity of the grid, which is conferred by its strict 
regularity or the parallelism of the beams in each way,

- the deformability of the individual members in bending which is linked to their slenderness.

The first condition is slightly flexible in practice $[4,5]$ but, theoretically, the kinematic condition for deployability of elastic gridshells is that the grid is made of equilateral quadrangles. Mathematically this is equivalent saying that the grid is a Tchebycheff net on the surface.

The most common technique to define such meshes on a given surface is the so-called compass method [2, 6]. Given a surface, a step length and two intersecting curves on this surface, it is theoretically possible to locally draw a Tchebycheff net starting from the point of intersection. This geometrical construction, which may be achieved with a compass, gives few control on the mesh and, until recently $[7,8]$, one could not say what surface can be meshed with the method. There are indeed some theoretical limitations linked with the curvature and the existence of a Tchebycheff net is only guaranteed for surfaces whose absolute Gaussian curvature is less than $2 \pi[8]$. Notice that another technique to find a mesh that fits a surface is to proceed as for material forming by using the finite element method to simulate the deformation of an elastic grid pressed on a surface [9]. Contrary to the compass method which is purely geometrical, this method has the advantage of including the bending stiffness of the members in the form-finding process.

The bottom-up approach proposed in this paper is another purely geometrical method. It relies on the duality between a certain family of circular meshes with a unique radius, called isoradial meshes in the following, and some Tchebycheff nets (see section 2.1). A circular mesh is composed of 
planar quadrilateral faces which are all inscribed in circles [10]. The interest of circular meshes for architecture has been shown, for example in [11] and relies on two key properties:

- they admit non-trivial offsets with a constant vertex height [10],

- circular meshes generated over a surface are discrete analogue of networks of principal curvature lines [12].

Moreover, it has been shown in [13], that, in many usual cases (such as structures under uniform normal loads) the curvature lines can be very close to principal stress lines, so that circular meshes provides a relevant lay-out for structural performance.

The proposed method allows for the design of a large variety of shapes from two curves in space. It is inspired by many design strategies which are abundantly used for architecture like translation surfaces [14], scale-trans surfaces [15], Monge and moulding surfaces [16], canal surfaces [17], etc. Actually, translation surfaces built from two curves with equal length subdivisions provide already a tool for the intuitive design of Tchebycheff nets with planar facets. However, their formal vocabulary is limited and, contrary to the proposed method, the associated PQ-mesh has no particular offset properties and is not linked with principal curvature direction which might lead to high curvatures in the members.

The paper is thus organised as follows. The first section gave a picture of the state-of-art on the morphogenesis of elastic gridshells. The second 70 section will detail the new methodology for the generation of isoradial meshes and their dual Tchebycheff net. Starting with one open directrix and one generatrix, the method will be extended to closed directrix and non-regular 
meshes with a strategy to deal with umbilical points. The third section will then illustrate these methods and provide an overview of the potential of the developed tools for surfaces from two curves. Section four will emphasise an alternative strategy for the treatment of singularities and section five will investigate the influence of the mechanical behaviour of the grid on the geometry of the Tchebycheff net and the planarity of the facets.

\section{Method for the generation of isoradial meshes and the associated Tchebycheff nets}

\subsection{Duality of isoradial meshes and Tchebycheff nets}

At the start of the proposed methodology, there is a remarkable observation on the duality between regular isoradial meshes (i.e. circular meshes with unique radius) and certain Tchebycheff nets. Indeed by joining the centre of each circle to the vertices of the quadrangle this circle circumscribes, one gets a quadrangular mesh in which the edges length are equal since all radius are equal. This generation process of the dual mesh of an isoradial mesh is illustrated on a plane example in Figure 1, but it works as well with circular meshes in space.

90

It must be noticed here that circular meshes constitutes a discrete equivalent of principal curvature network on smooth surfaces [18]. Hence the Tchebycheff net generated with this process goes through the curvature lines defined by the isoradial mesh and avoids thus the maximal curvature directions. Therefore, qualitatively, building an elastic gridshell with members following the lines of the Tchebycheff net, one would naturally minimise the bending stresses in the members. (Quantitatively, the precise evaluation of 


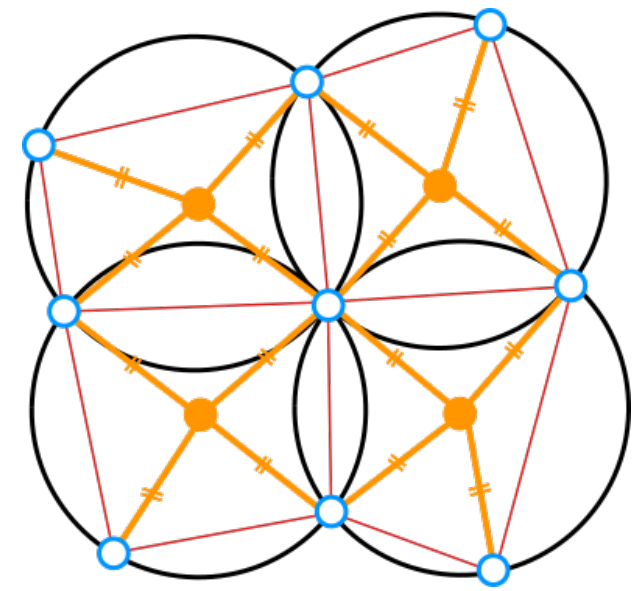

Figure 1: Local construction of a dual Tchebycheff net from an isoradial mesh.

the members curvature in the Tchebycheff net must take into account the geodesic curvature of lines which is not directly minimised by the generation process.) Furthermore, in the case of surfaces with positive Gaussian cur100 vature, this method offers a natural way to brace an elastic gridshell since the minimal curvature lines are immediately accessible through the isoradial mesh. In the case of surfaces with negative Gaussian curvature, the bracing is more complex, as the minimal curvature lines may have bending energy.

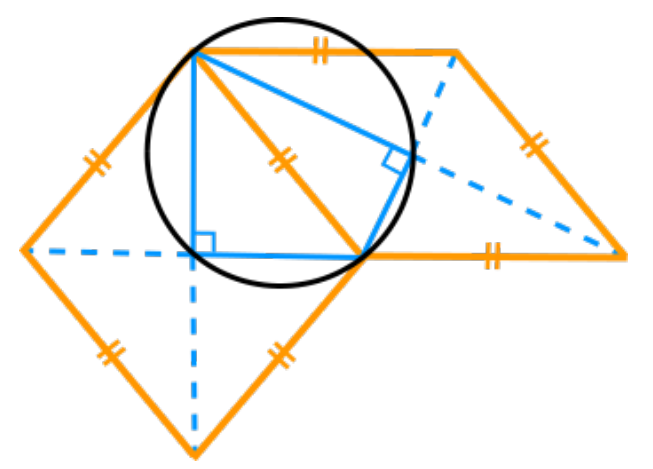

Figure 2: Local construction of a circular mesh from a planar Tchebycheff net. 
The duality described in the previous paragraph is even more peculiar corresponding design parameters. 


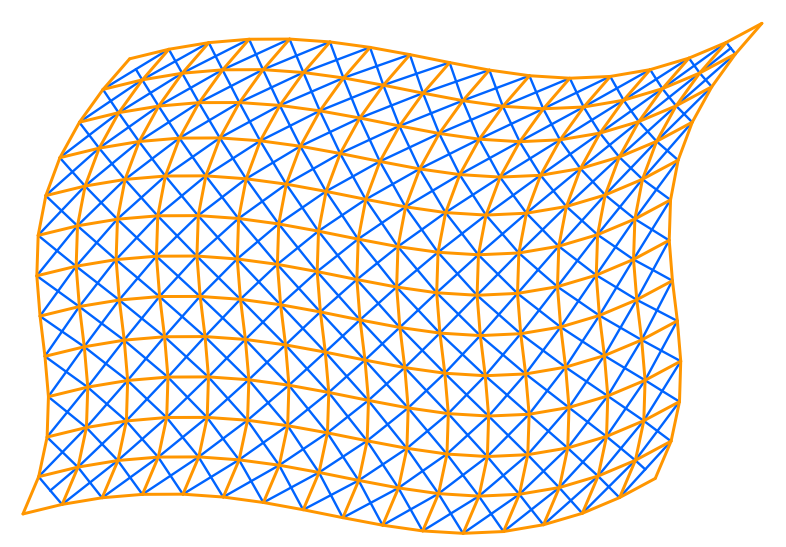

(a) Tchebycheff net and its diagonals

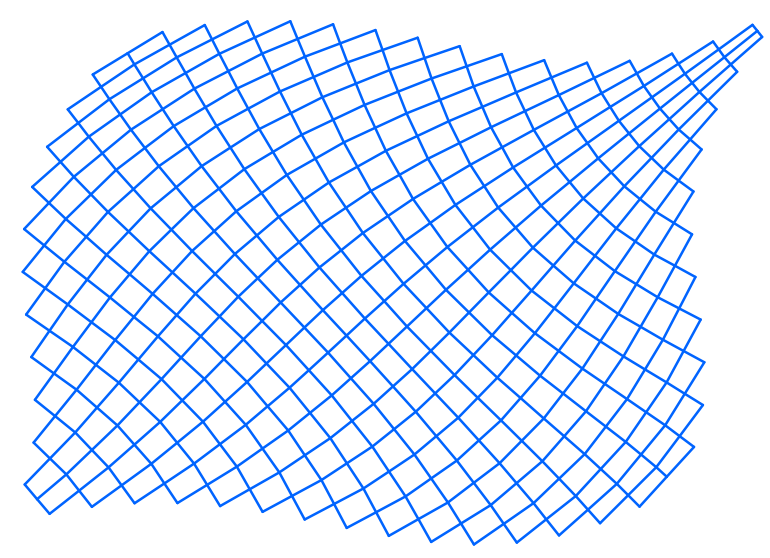

(b) Dual isoradial mesh

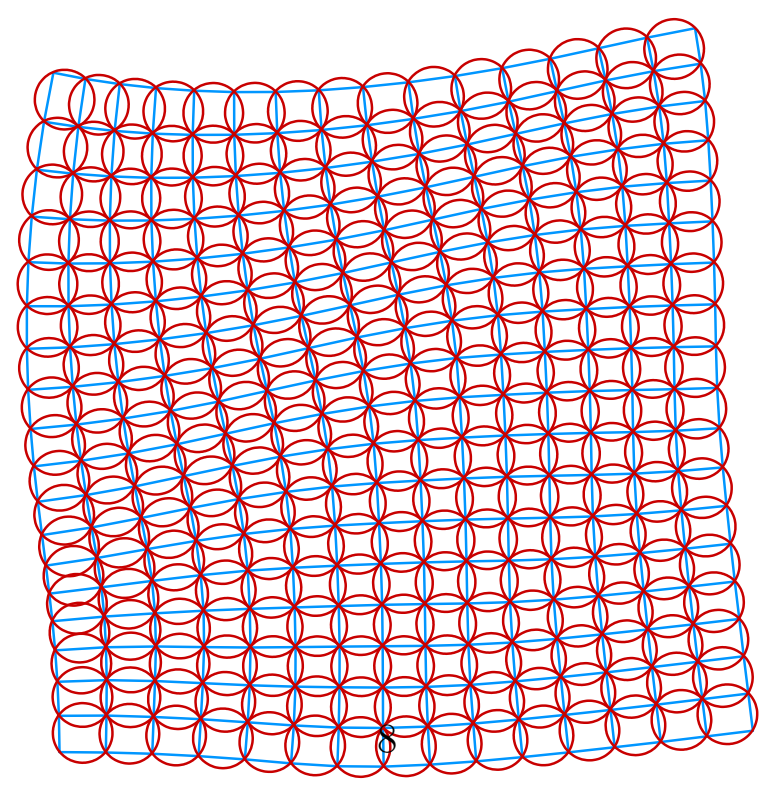

(c) Isoradial circles

Figure 3: Example of a dual isoradial mesh constructed from a planar Tchebycheff net. 


\subsubsection{Generation of a circular strip}

As explained in the introduction, the proposed methodology relies on the generation of a circular mesh along two curves: the generatrix and the directrix. To generate a circular strip, one must first define a subdivision of the directrix. This subdivision can be chosen arbitrarily, provided that the edge length is lower than the desired diameter $2 R$ of the circles. From the first two points $\left(\mathbf{P}_{0}^{0}\right.$ and $\mathbf{P}_{1}^{0}$ on Figure 4$)$ and the radius $R$, a torus can be generated. The intersection of this torus and the generatrix defines then the third point $\mathbf{P}_{0}^{1}$ and the first circle $\mathcal{C}_{1}$ (the practical calculation is detailed in section 2.2.3). The next circle $\mathcal{C}_{2}$ is then given by the intersection of the first circle $\mathcal{C}_{1}$ and the torus generated with the second and third points of the directrix, $\mathbf{P}_{1}^{0}$ and $\mathbf{P}_{2}^{0}$ respectively. And so on, until the last circle of the strip is generated. The fourth point on the last circle $\left(\mathbf{P}_{4}^{1}\right.$ on Figure 4$)$ can then be chosen freely provided that the quadrangle is not degenerated.

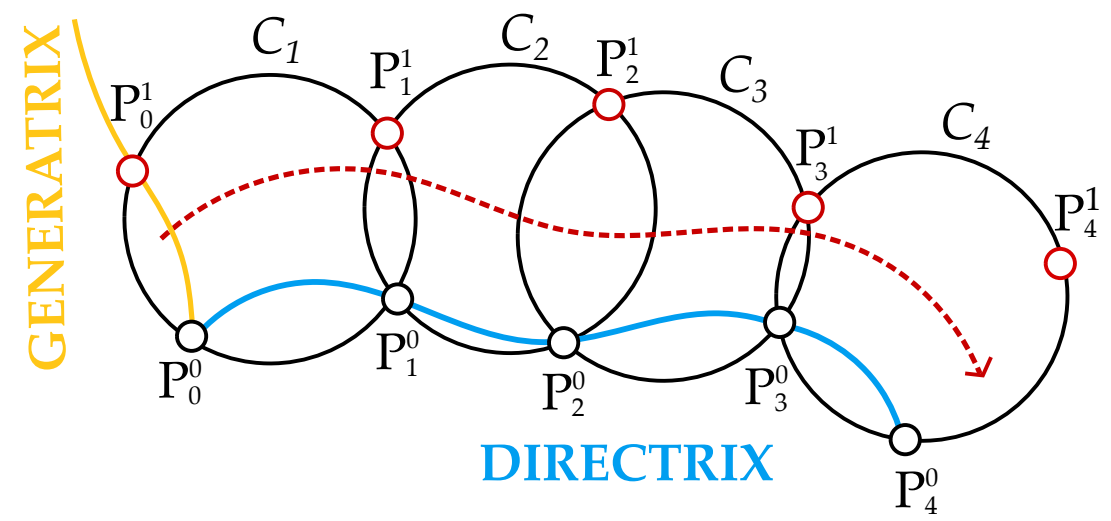

Figure 4: A strip of circles with the two rows of points (propagation from left to right).

It must be noticed here that the subdivision of the generatrix cannot be chosen arbitrarily but that it results from the construction method of the 
mesh and the successive intersection of the first circle of each strip and the

\subsubsection{Generation of a circular mesh}

Once the first strip has been generated, the curve defined by the second row of points constituted of points $\mathbf{P}_{i}^{1}$ can be considered as a new discrete directrix and the procedure which has just been defined can be repeated. And so on, until the algorithm stops, either because the whole generatrix is meshed or because the mesh cannot be propagated further for the chosen directrix subdivision and the chosen radius. It can be seen in Figure 5 that there is one degree of freedom on the position of the last point of the row in the first strip and that the location of this point has an influence on all the circles in the diagonal sector above it. This is directly related to the propagative nature of the mesh generation process. Particular care must thus be taken in the definition of the last points of each strip.

To satisfy the co-cyclicity constraint, the last point of each row must be on the last generated circle (the one passing through the last two points of the previous row and the penultimate one of the last). Its position is therefore controlled by one independent parameter. One may try to use this parameter to fit a third curve passing through the last point of the directrix in order to control the aspect of the end of the generated surface as well. The fitting would however be generally poor as the plane of the last circle in the row is determined by the propagation algorithm and is generally not equal to the tangent plane of the third curve. Imposing a less restrictive criterion is thus often preferable, like for example fitting a given plane passing through the last point of the directrix. Symmetry conditions are also particularly 


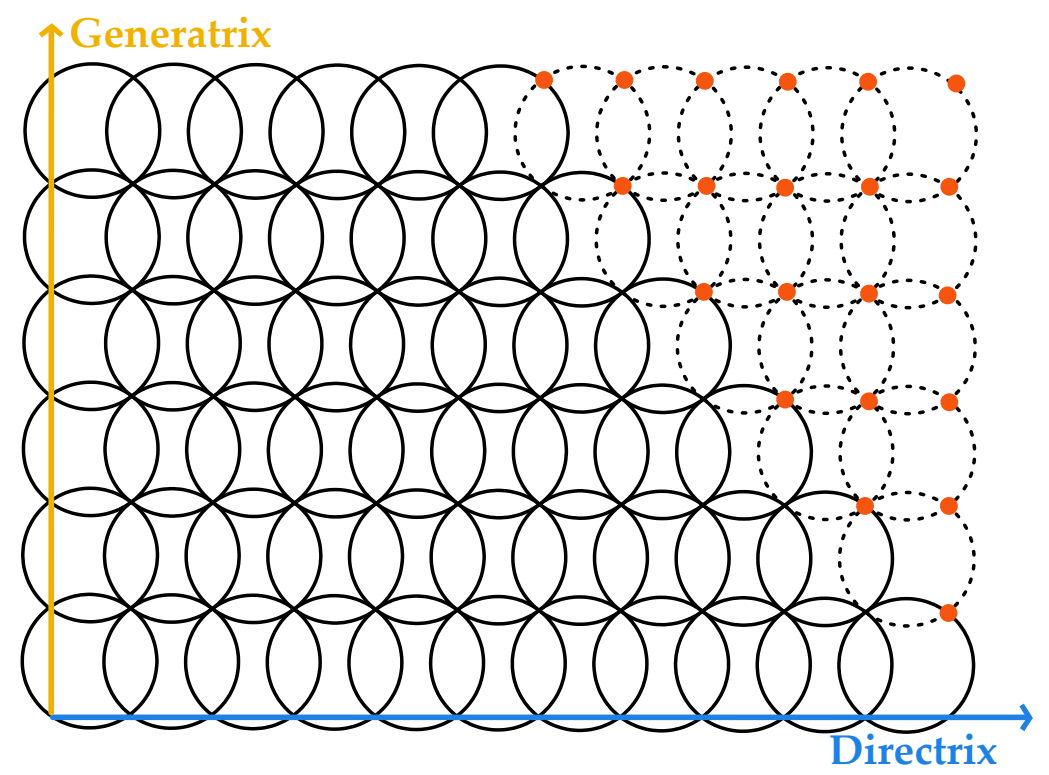

Figure 5: Areas of influence of the last point of the second row: circles affected (dashed lines) and circles independent (continuous lines).

interesting (see section 2.3.1). Another possibility is to consider that the last point of the row is defined to maximise the global smoothness of the final surface which can be evaluated with help of distortion energy (defined in section 4.1). This smoothness criterion can also be used to optimise the subdivision of the directrix.

\subsubsection{Practical generation of an intersecting circle of given radius}

The generation of an isoradial strip requires thus a method to generate a circle of given radius passing through two existing points. This is actually the main technical point of this method. It is depicted in Figure 6a. The circle known from the previous step $\mathcal{C}_{i}$ is drawn in light orange and the points $\mathbf{A}, \mathbf{B}$ and $\mathbf{C}$ on the directrix are shown in dark blue. We are looking for a 
circle $\mathcal{C}_{i+1}$ of radius $R$ which intersects twice $\mathcal{C}_{i}$ and which passes through the point $\mathbf{B}$ and $\mathbf{C}$ on the directrix. To this end, we first characterize which circles of radius $R$ go through $\mathbf{B}$ and $\mathbf{C}$ and then study among these circles which ones intersect twice the circle $\mathcal{C}_{i}$.

Family of circles with a given radius. The circles we are looking for go through $\mathbf{B}$ and $\mathbf{C}$ and have a given radius $R$. Consider now a point $\mathbf{P}$ on such a circle that is diametrically opposed to $\mathbf{B}$. The distance $\mathbf{B P}$ is equal to $2 R$ and basic geometry tells us that the triangle $\mathbf{B C P}$ is a right triangle. The locus of the points diametrically opposed to $\mathbf{B}$ is therefore the intersection of a sphere of radius $2 R$ centred in $\mathbf{B}$ and a plane normal to $\mathbf{B C}$ going through $\mathbf{C}$ (see Figure 6a). This is the circle $\mathcal{C}_{C}$ of radius $R_{\text {loc }}$ defined as follows:

$$
R_{l o c}=\sqrt{4 R^{2}-B C^{2}}
$$

Circle passing through a point and a given circle. The circle $\mathcal{C}_{i}$ and the prescribed point $\mathbf{C}$ on the directrix define a sphere (or a plane) $\mathcal{S}$. By definition, all circles intersecting twice the circle $\mathcal{C}_{i}$ and going through $\mathbf{C}$ belong to this sphere. Therefore the circle $\mathcal{C}_{i+1}$ is on $\mathcal{S}$ represented in Figure 6b).

Intersection of the two subsets. From the two preceding paragraph, it follows that the admissible circles can be defined by three points: $\mathbf{B}, \mathbf{C}$ and the point diametrically opposed to $\mathbf{B}$ which necessarily belongs to the circle $\mathcal{C}_{C}$ and to the sphere $\mathcal{S}$. The intersection of $\mathcal{S}$ and $\mathcal{C}_{C}$ is generally a set of two points which is not difficult to determine (indeed, the problem involving only spheres and circles, it can be simplified performing an inversion centred in $\mathbf{B}$ and by there quickly solved analytically). Among these two points, one can see on 


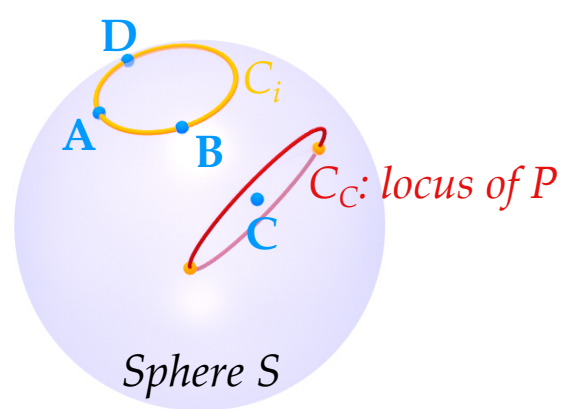

(a) Locus of circles family

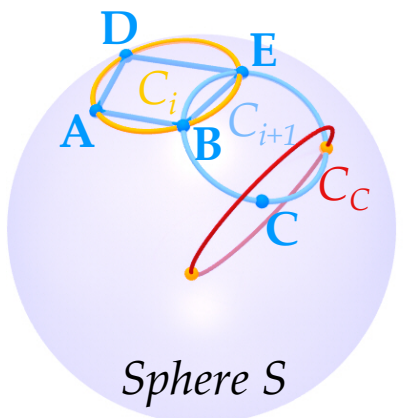

(b) Acceptable solution

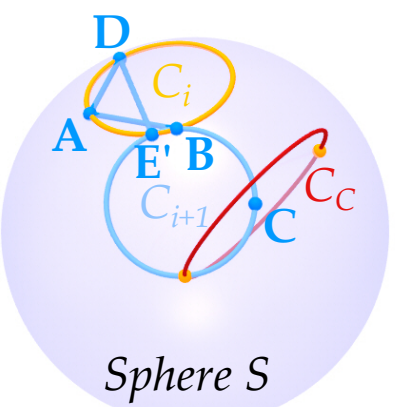

(c) Degenerated solution

Figure 6: Generation of an intersecting circle of given radius. 
Figure 6 that point $\mathbf{E}$ forms a convex quadrangle ADEB (Figure $6 \mathrm{~b}$ ) and point $\mathbf{E}^{\prime}$ forms a degenerated quadrangle $\mathbf{A D E} \mathbf{E}^{\prime} \mathbf{B}$ (Figure 6c). The convexity

of the quadrangle provides thus simple criterion for the algorithm to choose among the two points.

\subsubsection{Principle of the algorithm}

The algorithm steps are thus the following:

1. Define a subdivision of the directrix and a radius $R$ for the circles.

2. Construct the first circle of the strip from the first two points of the row and a third point on the generatrix.

3. Construct the next circle:

(a) Determine the points of intersection between the current circle and the two potential following ones, choose the circle that creates a convex quadrangle.

(b) If no intersection can be found, go back to iteration 1 .

4. Iterate 3 until the end of the directrix is reached.

5. Choose the last point of the row on it.

6. Iterate 2 until the end of the generatrix is reached.

220

An illustration of the shapes that can be generated with this method is shown in Figure 7. The subdivision of the directrix is here uniform and the choice of the last points in the row maximise the area of the last quadrilaterals. The dual Tchebycheff net is then obtained by dualisation, i.e. by 
joining the vertices of the isoradial mesh to the centre of each circle as in

Figure 1. Note here that since individual circular strips define curvature lines of the discrete surface, choosing the directrices in the normal plane of the generatrices will lead smoother and more intuitive meshes. Note also that this perpendicularity condition is however not necessary for the propagation algorithm to give valid isoradial meshes.

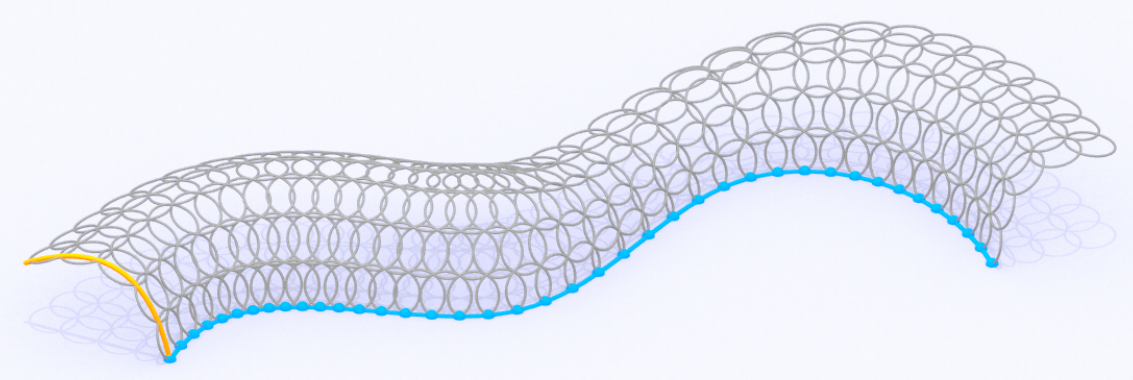

Figure 7: Generation of isoradial circles for given directrix (blue) and generatrix (orange).

\subsection{Generation of closed meshes}

The proposed methodology can be directly extended to closed directrix with a plane of symmetry. Its extension to any closed geometry requires however some modifications to insure a closing condition as will be seen in the following.

\subsubsection{Closed directrix with a plane of symmetry}

A curve with a plane of symmetry can be decomposed into two curves, one being the mirror image of the other. Considering this property and choosing 
one of these curves as open directrix and a curve in the plane of symmetry as generatrix, the algorithm of section 2.2 .4 allows for the generation of an isoradial mesh. Imposing for the choice of the last point of the row at step 5 that it is set as the second point of intersection of the last circle and the plane of symmetry, one can easily insure that the line formed by the last points of the row lays in the plane of symmetry. Joining then this isoradial mesh with its mirror image in the plane of symmetry, one gets a valid isoradial mesh built on a symmetric closed curve. A valid Tchebycheff net is also directly obtained by taking the dual of the isoradial mesh. Note that the directrix must not necessarily be planar. An example of closed dual meshes is shown in Figure 8.

\subsubsection{Closed directrix}

In the general case, the proposed propagation algorithm does not insure that the last circle of each strip intersects the first one and it must therefore be adapted. To this end, the cases where the first and last circles are cospherical and the other cases must be distinguished.

Cospherical circles. Indeed, if the first and the penultimate circles of the strip

are cospherical (possibly coplanar), every circle that intersects the first one also intersects the penultimate. The regular procedure for the construction of the next circle can thus be used to build the last circle of the isoradial strip. However, the choice of the fourth point of the last circle is not free but defined by the second point of intersection of the first and last circle. To insure the closing of the strip, the last point of the row must also be the first point of the row which can therefore not be defined freely at the beginning 


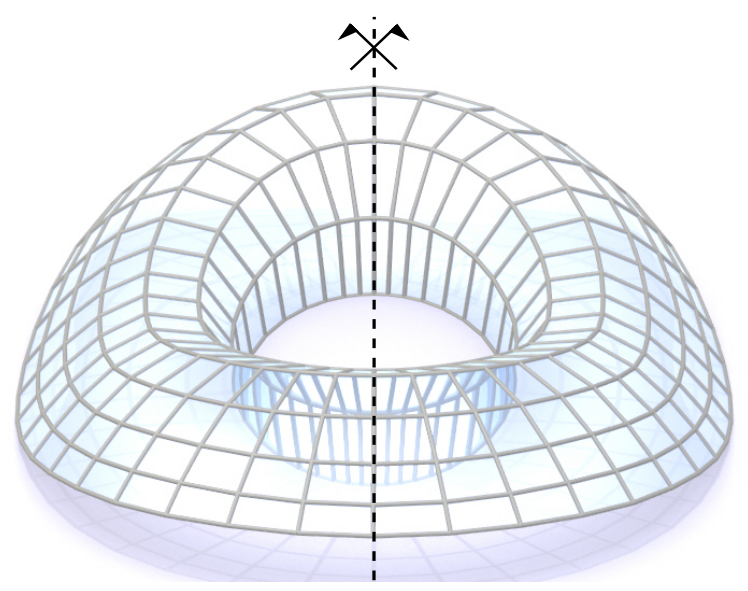

(a) Symmetric isoradial mesh

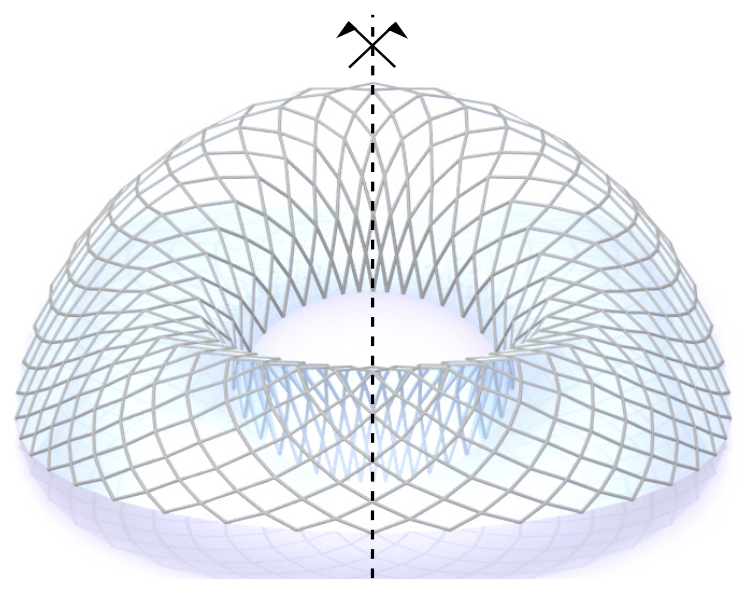

(b) Symmetric Tchebycheff net

Figure 8: Isoradial mesh and its dual Tchebycheff net with a plane symmetry. 
but only a posteriori. Practically, simply changing step 2 of the algorithm into "Construct the first circle of the strip from the first two points of the row and its centre so that its plane approximates the rectifying plane of the generatrix" insures hence the closing condition in this case.

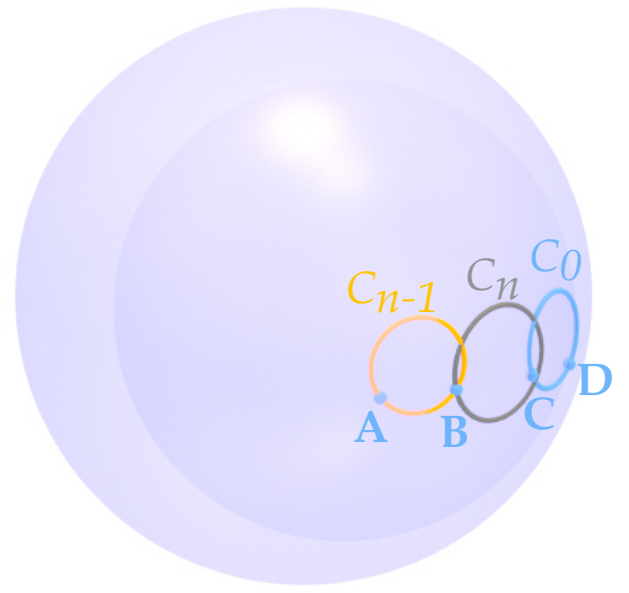

Figure 9: Design of the last circle of a closed strip as the intersection of two spheres.

Non-cospherical circles. In the case of Figure 9 where the first circle $\mathcal{C}_{0}$ and the penultimate circle $\mathcal{C}_{n-1}$ of the strip are not cospherical, the regular procedure can no more be used. Defining by $\mathbf{B}$ the last point of the row and $\mathbf{C}$ the first one, an easy way for constructing the last circle of the strip is to consider the sphere which contains $\mathcal{C}_{0}$ and $\mathbf{B}$, and the sphere which contains $\mathcal{C}_{n-1}$ and $\mathbf{C}$. By construction, the intersection of these two spheres is a circle $\mathcal{C}_{n}$ that passes through $\mathbf{B}$ and $\mathbf{C}$ and intersects both circles $\mathcal{C}_{0}$ and $\mathcal{C}_{n-1}$. The radius of the circle $\mathcal{C}_{n}$ is however arbitrary and therefore generally different from all the others. Like previously, the first and last point of the row are 275 again defined a posteriori and step 2 must be modified similarly by: "Con- 
struct the first circle of the strip from the first two points of the row and its centre so that its plane approximates the rectifying plane of the generatrix."

Limits and consequences on Tchebycheff nets. The solution proposed here for the construction of the closing circle creates a vertical strip of circles whose radii are different from the given radius of the rest of the mesh. First of all, these differences must not necessarily be visible as in the mesh illustrated in Figure 10 which is generated by a uniform subdivision of the directrix with a significant curvature. Then, considering the fact that the rationale of isoradial meshes is their link with Tchebycheff nets and the possibility that with planar quadrangular panels, this local irregularity is not necessarily prohibitive. Indeed, in terms of constructability of elastic gridshells, this vertical strip offers a well-defined area where beams lengths can be adjusted through a cut allowing the flattening of the whole grid (Figure 10). a closed directrix is general, but it might sometimes lead degenerated meshes where the radius of the last circle of a row becomes much larger than that of the rest of the mesh. This issue has been addressed in [20] who proved that parallel transport is linked to the notion of offset of polyhedral surfaces. In 295 practice, it is sufficient for the directrix to be quasi-spherical. This concept of advanced differential geometry describes a closed curve which is parallel to a closed curve on a sphere. Planar curves and curves with two planes of symmetry are simple examples of quasi-spherical curves. 


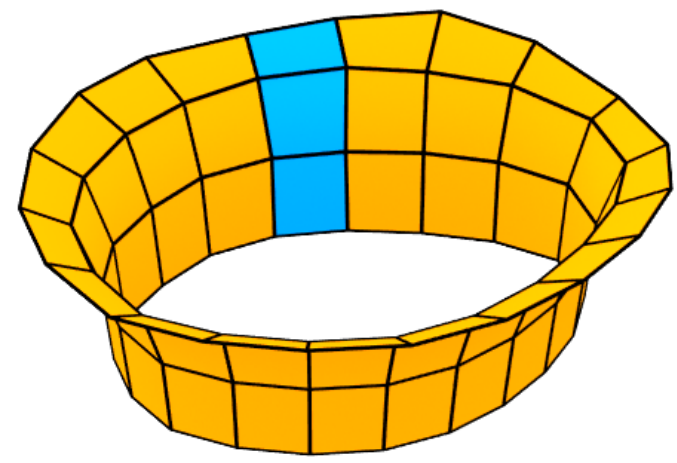

(a) Isoradial mesh

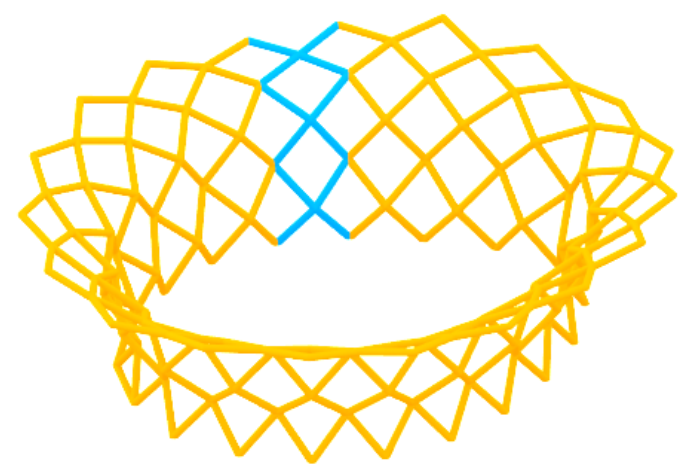

(b) Tchebycheff net

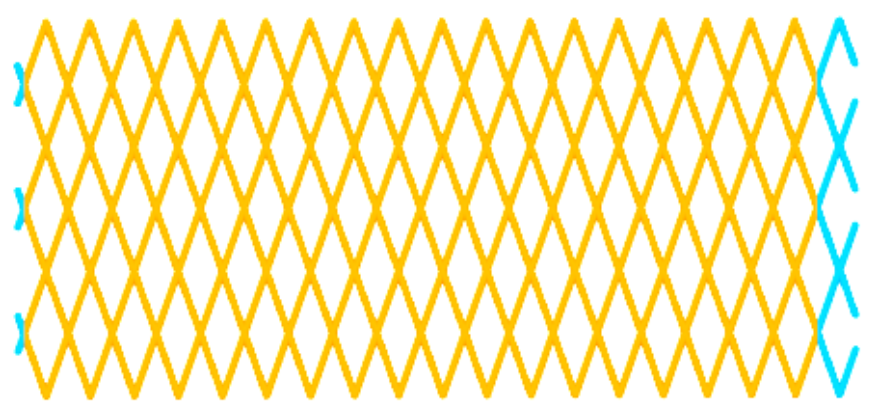

(c) Flattened Tchebycheff net

Figure 10: Vertical strip of circles of different radii (in blue) and their usage for the flattening of the associated Tchebycheff net. 


\subsection{Meshing singularities and umbilical points}

300 like tori constitutes a real enrichment of the formal universe of elastic gridshells, as none of the published built projects features closed loops. This formal universe can however be extended further, as the method can also be adapted to surfaces with umbilical points like the trinoid shown in Figure 11. is general and do not require symmetry.

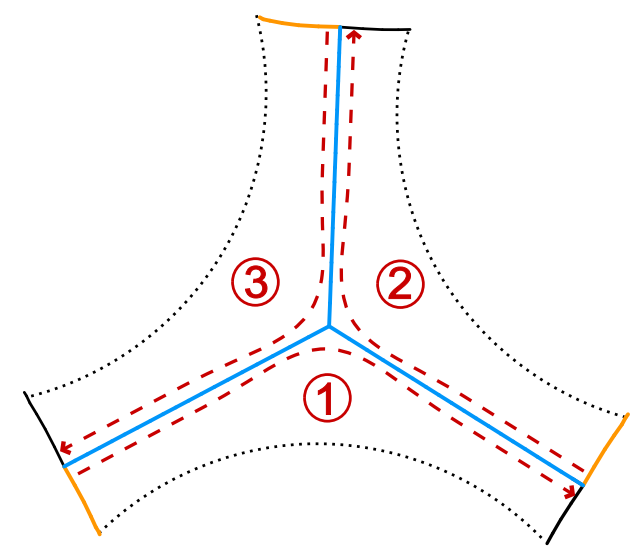

Figure 11: Generation of an isoradial mesh on a trinoid: directrices in blue, generatrices in orange.

The basic idea here relies on two colourability of meshes, i.e. the fact that the mesh can be filled with two colours like a checker board (see for example the mesh in Figure 12). Indeed, such meshes can be subdivided into one 310 independent family of strips (three strips in the example shown in Figure 11) [21]. Each strip can then be seen as an open mesh with a directrix along the common edges and a generatrix at the end of each strip. The compatibility of the meshes in the different strips is ensured by two issues: first the use of 
a unique radius for the whole mesh and then the use of the same subdivision of the directrix for each strip.

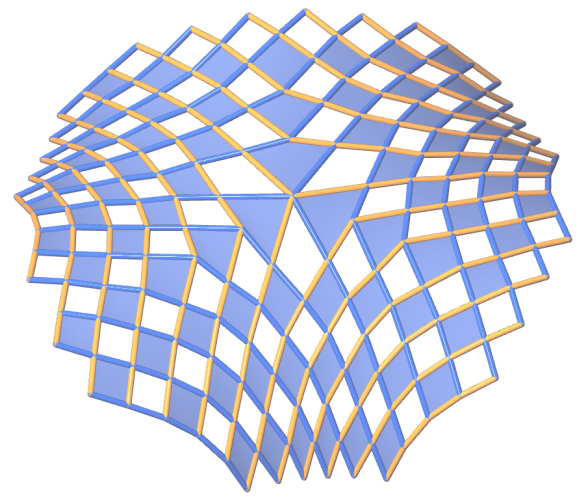

Figure 12: Two-colourable Tchebycheff net with a 6 -valence singularity: upper layer in orange and lower layer in blue.

From a constructional point of view, elastic gridshells are made of flat grids built with two superimposed layers. For the Tchebycheff nets in the different strips of a mesh with singularity to be compatible, it is thus necessary that consistent upper and lower layers can be assigned on the whole net. This compatibility condition is mathematically similar to the twocolourability condition (see for example the mesh in Figure 12). Therefore the two-colourability of the isoradial mesh ensures the buildability of the elastic gridshell from a two layers grid, except at the singularities where discontinuities in the members layer must be introduced through Tailor made connections. It is also worth remarking here that the resulting grid cannot be flatten as a whole. Therefore splitting strategies would have to be developped in further research to define subgrids that can be flatten, for example following the independant strips shown in Figure 11). 
An example of trinoid meshed with such a technique is shown in Figure 13. The valence of the central node or umbilical point is six. The three directrices are straight coplanar lines which are separated with an angle of $2 \pi / 3$. The generatrices are identical quarters of circle which are set in the bisecting plane of the directrices. Accounting for symmetries, meshing only one sixth of the total surface is sufficient, the rest is obtained by mirroring objects.

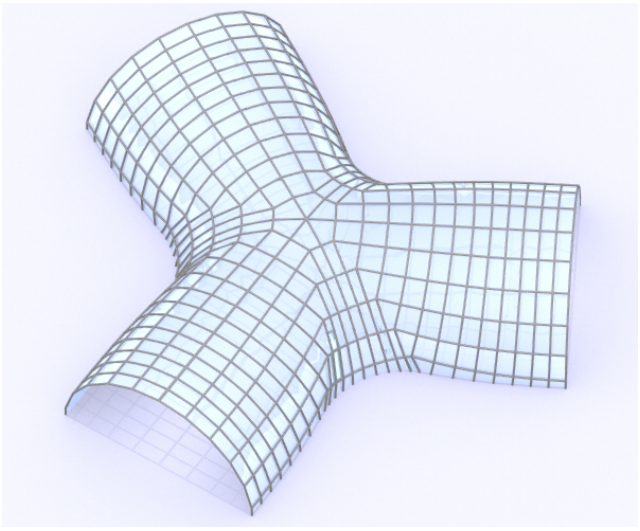

(a) Isoradial mesh

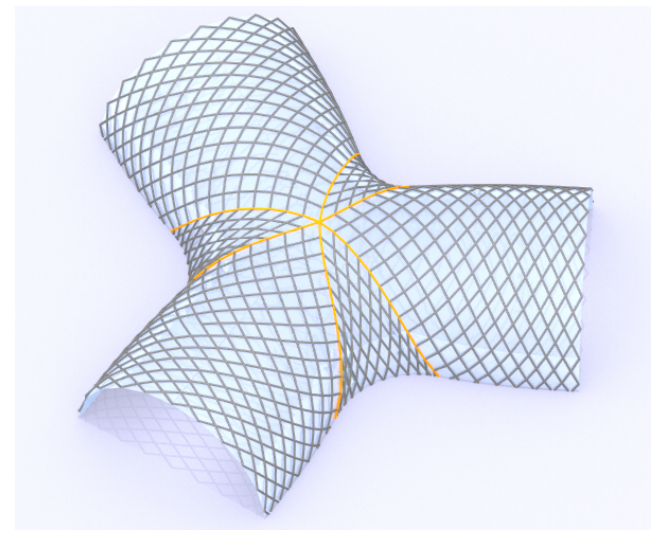

(b) Tchebycheff net

Figure 13: Valence-6 singularity with a regular subdivision. a singularity in the curvature or tangent of the directrix. This singularity is "propagated" along the diagonal of the mesh, creating six visible lines of distortion in the mesh with rhombic facets in the isoradial mesh. (The meshes remain exact isoradial and Tchebycheff meshes, although they are not smooth.) These lines of distortion in the isoradial mesh correspond to curvature concentration in the Tchebycheff net whose lines change suddenly direction when they cross the diagonals (see orange lines in Figure 13b). The curvature concentration might be inacceptable in practice for the realisation 
of an elastic gridshell and therefore an alternative strategy to smoothen the net has been developed in section 4.1.

\section{Surfaces from two curves}

\subsection{Shape exploration}

The algorithm described in the previous section allow us to generate surfaces defined by two curves: a generatrix and a directrix. Although very simple, this propagation method has a large potential for the generation of usual shapes of elastic gridshells, as illustrated by Figures 14 and Figure 15. The first ones shows a structure inspired by Edward Cullinan's Weald and Downland Gridshell built in 2002 [22, 23] and the second a structure similar to the roof by Glen Howells' Savill Building built in 2006 [23, 24]. The method not only reproduces the lay-out of the existing wooden grid but it supplies a lay-out for its covering with planar quadrangular facets which might have been of interest in one case or the other. The pattern created by the Tchebycheff net is far from obvious and would be hard to obtain with the compass method on the smooth surface. This illustrates the interest of using the dual of the Tchebycheff net for the construction of the surface.

Shifting now to closed directrix, the algorithm is proved to be quite efficient for the design of stadium. The one represented in Figure 16 is inspired by Jean-Michel Wilmotte's football stadium, the Allianz Riviera in Nice, France. The actual structure is not an elastic gridshell but a rigid one whose

wooden structure is well represented by the Tchebycheff net on the right, while its ETFE envelop could be efficiently replaced by planar quad cover based on the circular mesh shown on the right. Domes with oculus can also 


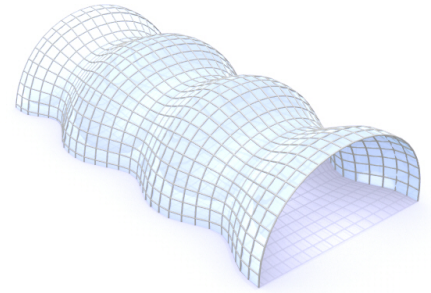

(a) Main structural grid

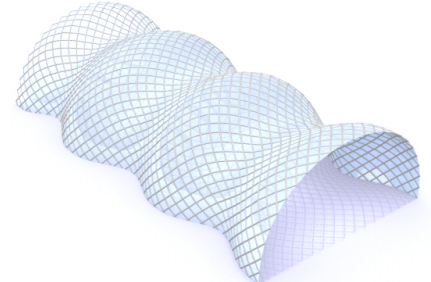

(b) Cover with planar quadrangular panels

Figure 14: Elastic gridshell inspired by the Weald and Downland Museum, UK.

Figure 15: An elastic gridshell inspired by the Savill Building, UK. 
be designed with this process. The one represented in Figure 17 is inspired by Denis Montel's domes designed for Hermès in Paris, France in 2010. The 370 realisation of these "basket" structures was of huge complexity [25] and could have been simplified if an elastic gridshell solution had been explored.

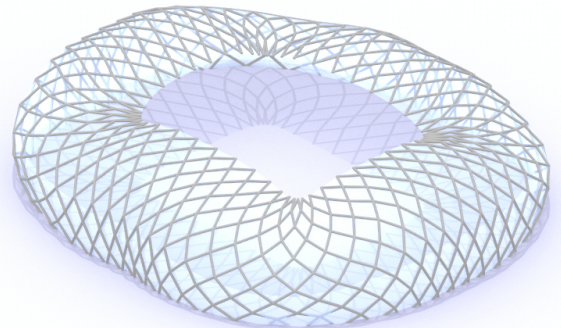

(a) Main structural grid

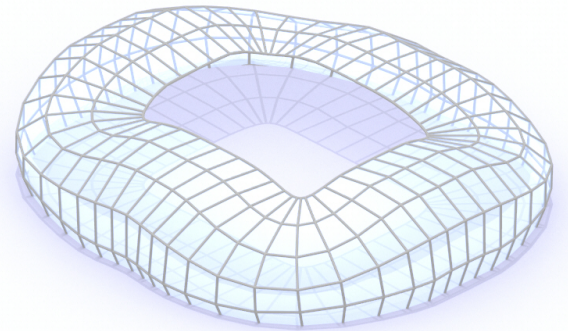

(b) Cover with planar quadrangular panels

Figure 16: Stadium inspired by the Allianz Riviera in Nice, France.

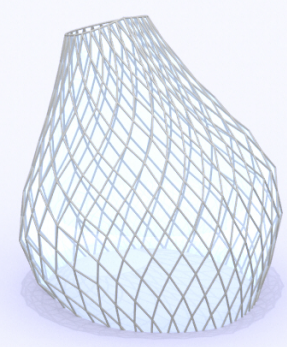

Figure 17: Dome with occulus.

\subsection{Remeshing}

The mesh size of an elastic gridshell might be subject to changes over the course of a project, due to mechanical or constructive requirements. It is therefore necessary to be able to change the mesh size without changing 
the overall shape, which is set during early stages of the project. In the proposed method, the generation of Tchebycheff nets proceeds from its dual, from the setting of the subdivision of the directrix and the reference radius. Yet, it can be remarked that this is equivalent to fixing the angles between the Tchebycheff nets and the directrix. Indeed, one can see on Figure 18 that, once the radius is fixed, it is equivalent to set the points $\mathbf{A}_{\mathbf{i}}$ or the angles $\alpha_{i}$.

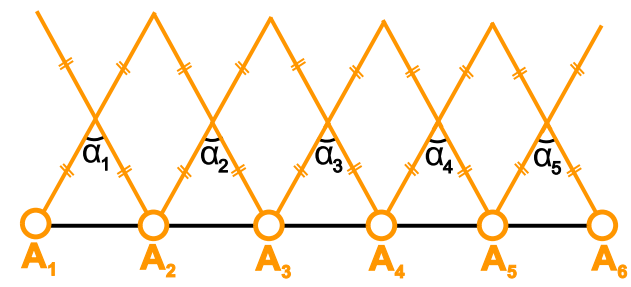

Figure 18: Angles of a Tchebycheff net.

Both sets of variables are linked by equation 2 which evidences the fact that, for a given subdivision of the directrix, an increase in the radius will decrease the angles of the Tchebycheff net.

$$
\sin \frac{\alpha_{i}}{2}=\frac{A_{i} A_{i+1}}{2 R} .
$$

This relation between the angle of the net and the subdivision of a line of the dual is at the basis of the method used by [8] for the proof of the meshability of surfaces by Tchebycheff net. In the tools developed here however, the points on the directrix remain the primary variables and the angles secondary variables with no direct control by the user.

From equation (2), it appears hence that, varying simultaneously the radius and the edge lengths so that the characteristic angles of the Tchebycheff 
net is constant, will let the underlying smooth surface unchanged. The isoradial meshes generated with this constraint will all be discrete analogue of curves will lead to a discretisation of the same surface.

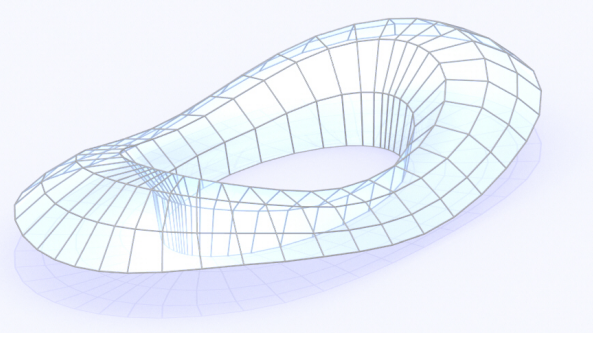

(a) Coarse mesh

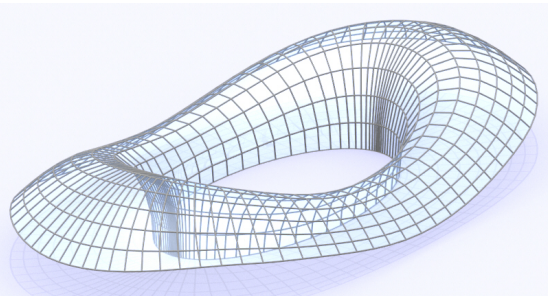

(b) Dense mesh

Figure 19: Two isoradial meshes generated from the same generatrix and directrix with the same value of $n \cdot R$.

\subsection{Curvature and subdivision of the directrix}

Looking now at Figure 20, it is remarked that, in some areas, quadrangles on the last strip have become very flat which indicates that the angle between the two directions of the Tchebycheff net is close from $\pi$ (this issue is wellknown in practice from users of the compass method). As a matter of fact, the mesh could not be propagated further: the algorithm had stop at that row. It is also remarked that these area belong to curvature lines which start in 
the portions of the directrix where the curvature are the highest. Yet the fact that the meshability of a surface is linked to its total curvature is well known and we are facing here the reverse problem as we are generating the surface together with the Tchebycheff net on it. The proof of this assertion is beyond the purpose of this article and would need further researcher. However we will show that in practical cases this problem can be dealt by adapting the mesh density to the curvature of the directrix.

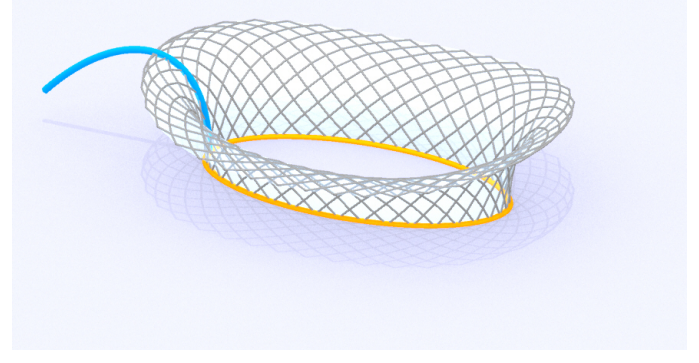

Figure 20: Tchebycheff net limited by the flattening of quadrangle.

Consider for example Figure 20, the directrix is an ellipse and the generatrix is a parabola. The curvature variations of the directrix are substantial and its subdivisions are uniform. It is noticed that the algorithm cannot mesh the whole generatrix and stops because rhombi in areas of high curvature tend to become flat.

A first strategy to overcome this problem will be to increase the reference radius so that, according to equation (2), the starting angles of the Tchebycheff net will be sharper, postponing hence the moment where they become flat and allowing the algorithm to mesh the whole generatrix. This 425 however will not change the fact that uncontrolled distortion in the mesh 
will appear in the area of high curvature. A second strategy is thus proposed here by meshing the directrix with edge length inversely proportional to the curvature as shown in Figure 21).

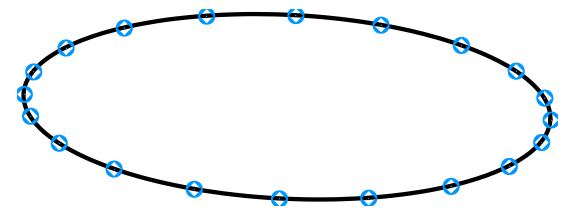

(a) Uniform subdivision

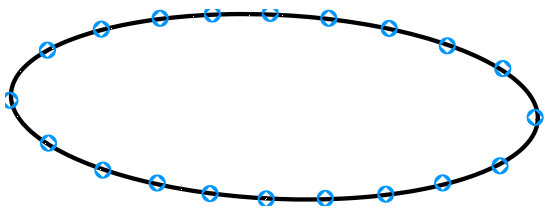

(b) Subdivision with curvature

Figure 21: Uniform and non-uniform subdivisions of an ellipse.

Generating then the Tchebycheff net based on this new subdivision, one obtains Figure 22. The generatrix is completely meshed and the mesh looks uniform and regular. Moreover, the overall shape of the mesh resemble what one would have expected generating a surface by sweeping the generatrix along the directrix in a $3 \mathrm{~d}$-modeller. This strategy seems thus to lead intuitive results in terms of shape generation and is therefore recommended.

Figure 22: Tchebycheff net based on the same data as Figure 20 with a subdivision adjusted to the directrix curvature. 


\section{Optimisation around umbilical points}

It has been seen in section 2.4 , that the curvature discontinuity of the directrix around an umbilical point is propagated diagonally through the mesh (cf. Figure 13) which causes:

- diagonal sets of distorted panels in the isoradial mesh (see hatched quadrangles on Figure 23);

- curvature discontinuities in one direction of the Tchebycheff net which is actually the dual counterpart of the local distortion of the mesh.

From a constructional point of view, this curvature singularity corresponds to unacceptable bending stresses in the members of the elastic gridshells.

445 An alternative strategy based on mesh relaxation is thus proposed in the following.

\subsection{Mesh relaxation}

The strategy of mesh relaxation consists in opening a hole around the singularity by disconnecting locally the strips of the mesh. Doing so, the directrices become smoother and valid isoradial and Tchebycheff meshes can be generated on each strip. For example, in the case shown in Figure 11 with a single singularity and three independent strips, one might chose to disconnect three points on each branch plus the umbilicus point (see blue dots in Figure 23) and to relax the mesh to remove distorted panels. A temporary change in the mesh topology is hence accepted during the mesh relaxation phase, the initial topology will be recovered afterwards during the hole filling phase described in section 4.2.

To evaluate the relevance of this strategy, the quality of the generated 


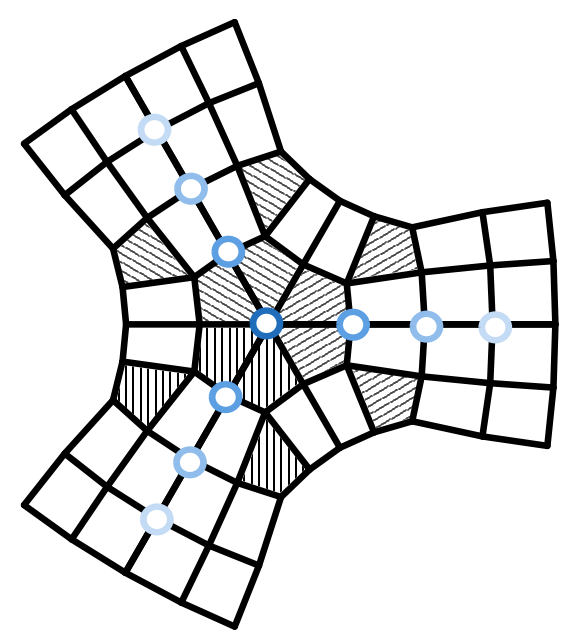

(a) Initial mesh (distorted panels hatched)

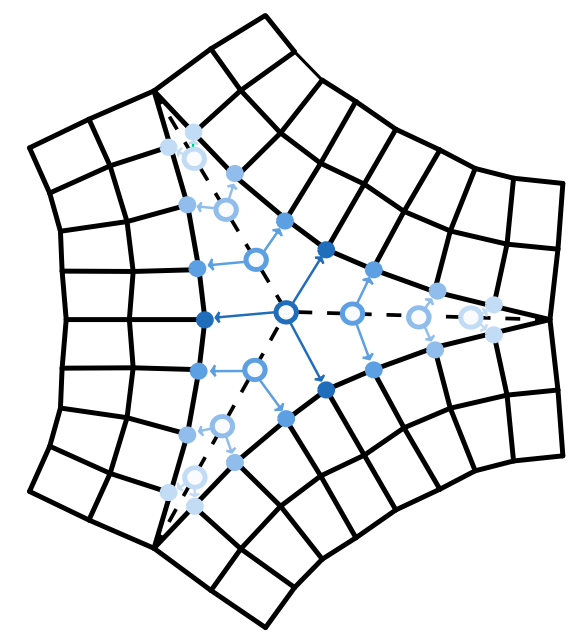

(b) Relaxed mesh

Figure 23: Mesh relaxation by local opening around the umbilicus.

mesh should be estimated. This could be done through the bending energy of the Tchebycheff net given by equation 5 or through a kind of distortion energy of the isoradial mesh. This energy $E_{d}$ measures the gap between the current quadrangle and a rectangle through its actual angles $\alpha, \beta, \gamma, \delta$ :

$$
E_{d}=\left(\alpha-\frac{\pi}{2}\right)^{2}+\left(\beta-\frac{\pi}{2}\right)^{2}+\left(\gamma-\frac{\pi}{2}\right)^{2}+\left(\delta-\frac{\pi}{2}\right)^{2}
$$

As the quadrangles are all inscribed in circle, opposite angles are supplementary and the expression of this distorsion energy reduces to:

$$
E_{d}=2\left(\left(\alpha-\frac{\pi}{2}\right)^{2}+\left(\beta-\frac{\pi}{2}\right)^{2}\right)
$$

From our experience, it results that minimising the distortion of the circular mesh minimises also the bending energy of the Tchebycheff net while the contrary is not necessarily true. For this reason, comparisons of the mesh 
performances in the following will be done on the distortion energy in the mesh.

To determine what the optimum size of the opening is, four symmetric options have been tested, varying the number of disconnected points in the area of the singularity. Each time, the distortion energy is minimised varying the positions of the disconnected points in the plane of the singularity. The algorithm for minimisation is here BFGS. The resulting optimal configurations are then compared in Figure 24. It can be seen that it decreases significantly with the number of points but reaches a plateau around 7 displaced points (the case illustrated in Figure 23 corresponds to 10 displaced points: 3 in each sector plus the singularity). For this configuration, the distortion energy has been divided by 150 compared to the energy of the mesh without hole $E_{d}^{*}$. The regularization strategy is thus efficient and local, as few points are necessary to drastically improve mesh quality.

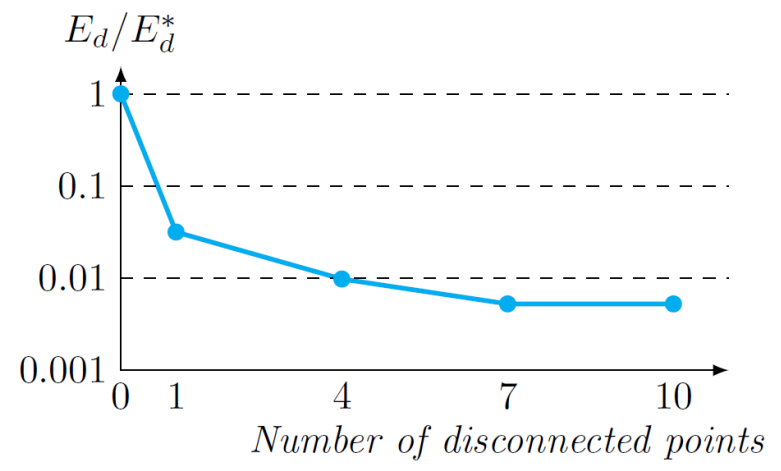

Figure 24: Normalised distortion energy with the number of disconnected points. 


\subsection{Hole filling}

The strategy proposed in the preceding section leads to the opening of a polygonal hole in the mesh around the umbilical point (see Figure 23)

485

490 1. Choose the width of the hole and minimise the distortion energy as in

which needs to be filled. To this end, like for closed directrix in section 2.3.2, we propose a solution for the hole filling which locally violated the isoradial condition of the mesh (actually the panels in the neighbourhood of the umbilicus are not even circular). This solution consists in three successive optimisations: section 4.1 .

2. Reconnect the points along the directrices and optimise their positions in space, so that the quadrangle tends to planar facets (coloured panels on Figure 25).

3. Optimise the location of the crossing points of the Tchebycheff net in those panels, so that the bending energy of the Tchebycheff net is minimal.

The first optimisation has just been presented in previous section. The second one is an optimisation over the coordinates of the reconnected points in order to minimise the planarity energy of the mesh which is here evaluated from the volume of the tetrahedra formed by each face. It appears in Table 1 that this energy tends to increase with the number of disconnected points (or with the width of the hole). The warping of the panels remains however satisfactory and the mesh can be called a PQ-mesh. 


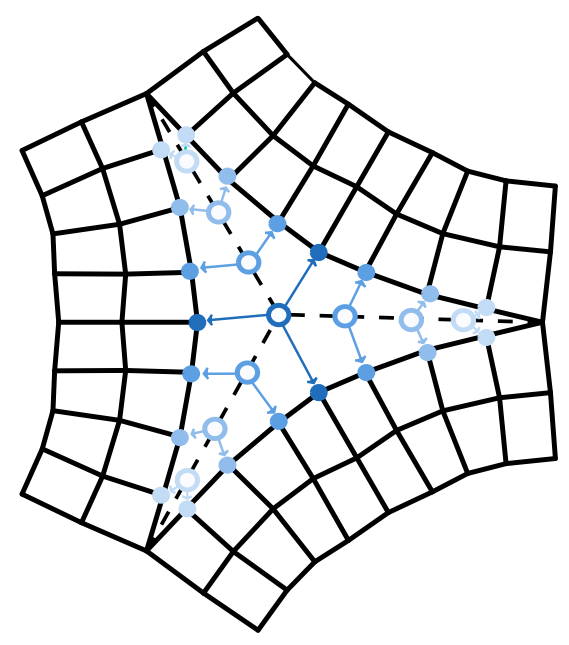

(a) Relaxed mesh

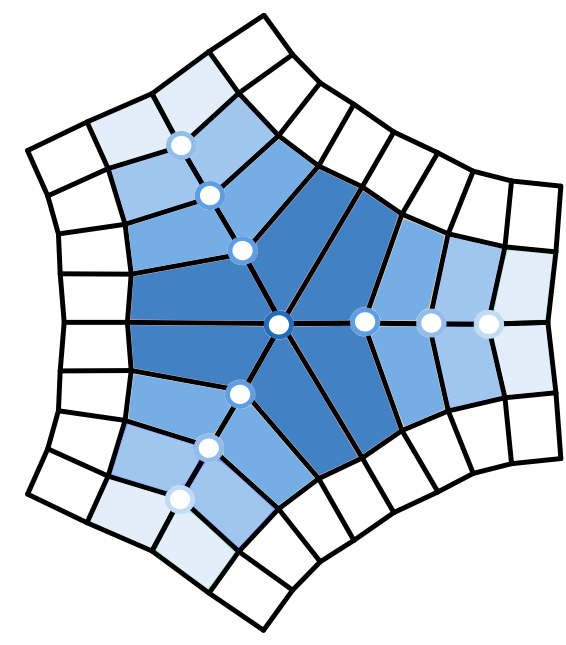

(b) Final mesh

Figure 25: Reconnection of the points in the area of the singularity after mesh relaxation.

\begin{tabular}{|c|c|c|}
\hline $\begin{array}{c}\text { Number of } \\
\text { points }\end{array}$ & $\begin{array}{c}E_{\text {planarity }} \\
\text { before optimisation }\end{array}$ & $\begin{array}{c}E_{\text {planarity }} \\
\text { after optimisation }\end{array}$ \\
\hline 1 & $4 \cdot 10^{-3}$ & $2 \cdot 10^{-5}$ \\
4 & $7 \cdot 10^{-2}$ & $2 \cdot 10^{-3}$ \\
7 & $5 \cdot 10^{-2}$ & $1 \cdot 10^{-2}$ \\
10 & $5 \cdot 10^{-2}$ & $8 \cdot 10^{-3}$ \\
\hline
\end{tabular}

Table 1: Comparison of the mean planarity energy in function of the number of disconnected points. 


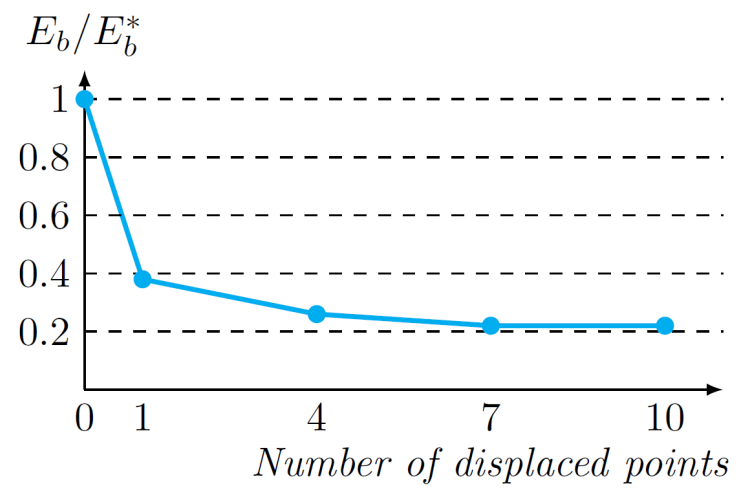

Figure 26: Variation of the normalised bending energy of the Tchebycheff net with the number of disconnected points in each sector.

In the third optimisation, the points corresponding to the centre of the circle in the regular mesh are created in the non-circular quadrangles and their positions in space is chosen, so that the bending energy of the now "quasi-Tchebycheff" net is minimum. This energy is given by equation 5 and decreases significantly with the width of the hole (see Figure 26). The gain with this last optimisation is of approximately 5 in the present example. The results of these three optimisations for the example of Figure 13 is shown in Figure 27.

\section{Mechanical optimisation of the Tchebycheff net}

In the preceding sections, the dual Tchebycheff nets were generated from the vertices of the isoradial meshes and the centre of the corresponding circles. Although this theoretically allows for the realisation of the gridshell from a planar two-way grid, the final form of the Tchebycheff net does not correspond to a state of mechanical equilibrium. On the contrary, the proposed procedure for the generation of the Tchebycheff net leads to stress 


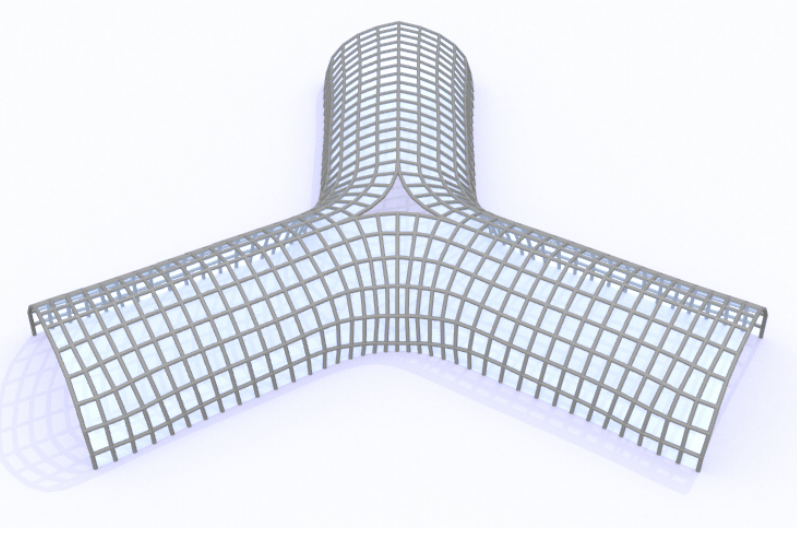

(a) Mesh relaxation

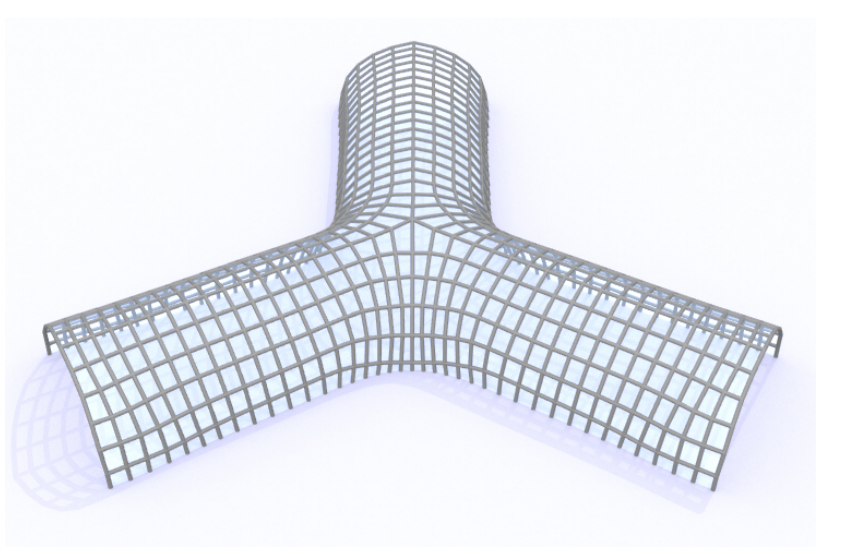

(b) Hole filling and planarity optimisation

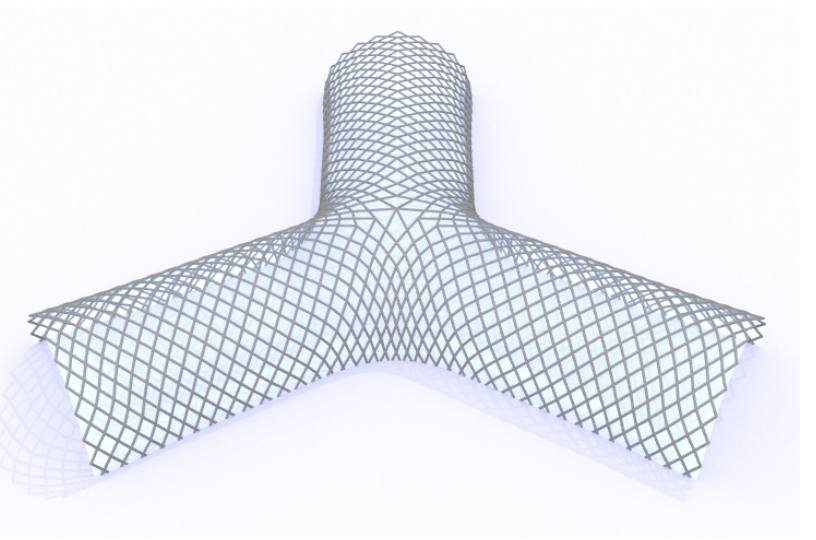

(c) Bending energy optimisation

Figure 27: Mesh relaxation and hole filling of a valence-6 singularity. 
520 mesh and change faces. Indeed, one can see on Figure 28 (which represents the bending moments in the mesh shown in Figure 7) a clear alternation of high and low values of the bending moments in the beam.

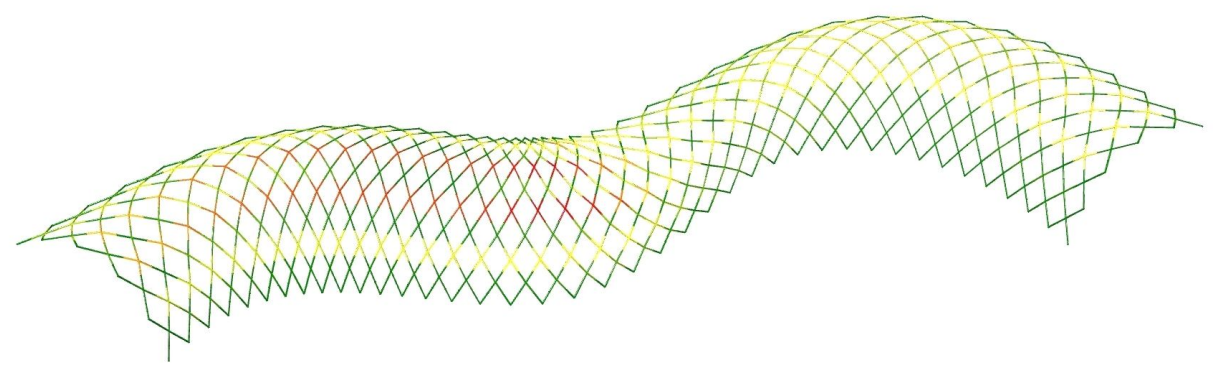

Figure 28: Bending moments in the Tchebycheff net directly derived from the isoradial mesh of Figure 7 (high values in red and low in green).

To overcome this sub-optimal configuration from a mechanical point of view, three strategies can be implemented:

- a purely geometrical offset of circle centres,

- a mechanical offset with priority to the planarity of the faces,

- a mechanical offset with priority to the regularity of the grid.

The three strategies will be developed in the following subsections and finally

compared in terms of bending energy of the Tchebycheff net or planarity of the panels. The bending energy of the Tchebycheff net measures the discrete curvature of the lines. It can be defined as the sum of the square of the angles $\alpha_{i}$ made by successive segments of the Tchebycheff net:

$$
E_{b}=\sum \alpha_{i}^{2}
$$

In practical cases where the structure is made of circular tubes (like for 
535 energy of the grid members: it has thus the same minima.

\subsection{Geometrical offset}

The regularity of the Tchebycheff net comes from the fact that every segment of the grid has the size of the circle radius. However, moving the circle will also lead to valid Tchebycheff nets ( $c f$. Figure 29). An infinity of dual Tchebycheff nets can hence be associated to a single isoradial mesh. Considering the fact that these Tchebycheff nets can be used as a reference geometry for the practical realisation of elastic gridshells, it is interesting to associate a bending energy to the net and to optimise the offset $\mathbf{O O}^{\prime}$, so that this bending energy is minimal.

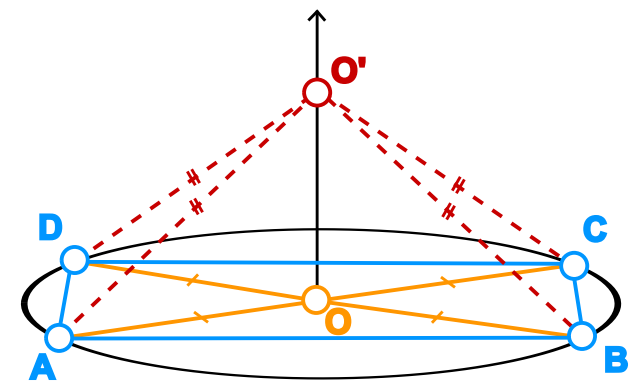

Figure 29: Offset of the Tchebycheff net along the normal.

The offset being done along the normal of each circle, its direction lines are completely defined by the isoradial mesh. Its value can be chosen freely, but must be constant along the mesh to ensure that the dual mesh is a Tchebycheff net. The relative value is however unconstrained and we choose to set it locally according to the mean curvature. To this end, the two principal 
curvatures in the neighbourhood of the circle are evaluated and compared. Then the local value of the offset is defined, so that the Tchebycheff net "pops up" in the direction of the highest curvature.

In practice, considering a common circle of the net $\mathcal{C}_{0}$ and its four neighbour $\mathcal{C}_{1}$ to $\mathcal{C}_{4}$ on Figure 30, the two principal direction are defined by the strip $\mathcal{C}_{1}, \mathcal{C}_{0}, \mathcal{C}_{3}$ and the strip $\mathcal{C}_{2}, \mathcal{C}_{0}, \mathcal{C}_{4}$. The mean curvature in one direction can be defined as the average radius of the two spheres containing $\left[\mathcal{C}_{1}, \mathcal{C}_{0}\right]$ and $\left[\mathcal{C}_{0}, \mathcal{C}_{3}\right]$. The evaluation of the radii of these sphere is straight forward, considering two successive circles, both of radius $R$ (see Figure 30). Their intersection defines an angle $\beta$ between their tangents and an angle $\alpha$ between their normals. The radius $r$ of the sphere containing these two circles is given by:

$$
r=R \sqrt{\frac{1+\cos \beta}{1-\cos \alpha}}
$$

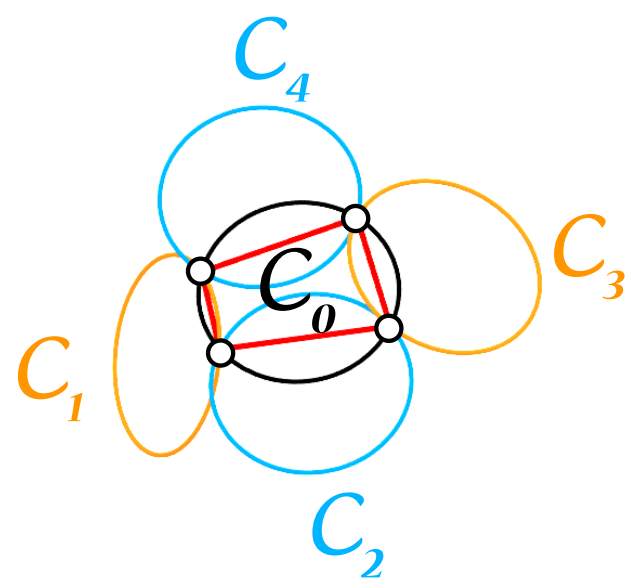

(a) Neighbouring circles

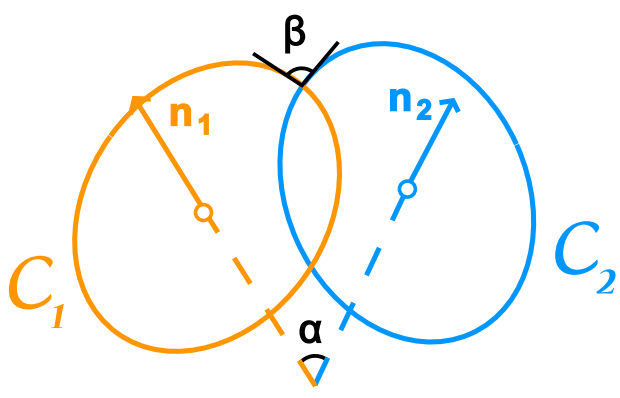

(b) Characteristic angles

Figure 30: Determination of the curvature in the neighbourhood of a circle.

Once the relative direction of each offset as been locally defined, the 
565

minimised. The resulting length increase $\Delta l_{\text {geom }}$ is easily deduced from the offset value $h$ by Pythogora's theorem:

$$
\Delta l_{\text {geom }}=\sqrt{1+(h / R)^{2}}-1
$$

\subsection{Form-finding with priority to the cover planarity}

The second strategy is inspired by the construction process of some recent 570 projects $[22,24,29]$ where the grid has been assembled on a gigantic scaffolding while its elastic deformation was monitored with hundreds of props. In those cases, the final form is obtained by bracing the grid while it is still lying on the scaffolding, locking in the stresses induced by the props. The removing of the scaffolding then does not alter significantly the form as it is

fixed by the bracing. With this complex process, the designer can impose a form to the grid.

Supposing hence that a prop is set at each vertex of the isoradial mesh, one can impose the form of the mesh to the grid. Yet to diminish the bending moments in the members, one can imagine a form-finding step based on these boundary conditions where the member lengths are unknown and driven by the bending forces. This is easily done with the dynamic relaxation method by arbitrary lowering the axial stiffness of the members and relaxing the mesh in a similar way to what was done in [30]. Once the equilibrium has been reached, a new grid can be defined based on the lengths in the final 585 configuration. This grid is irregular, but, tuning the relative bending/axial stiffness, this irregularity can be kept sufficiently low, so that in practice the grid can be easilly assembled as demonstrated in [5]. After this form-finding 
step, one gets hence a slightly irregular grid with smooth bending moments (see Figure 31) supporting a cover with perfectly planar quadrangular panels. between the maximal length $l_{\max }$ and the average length which is equal to the isoradial mesh radius $R$ :

$$
\Delta l_{\text {planar }}=\frac{l_{\max }-R}{R}
$$

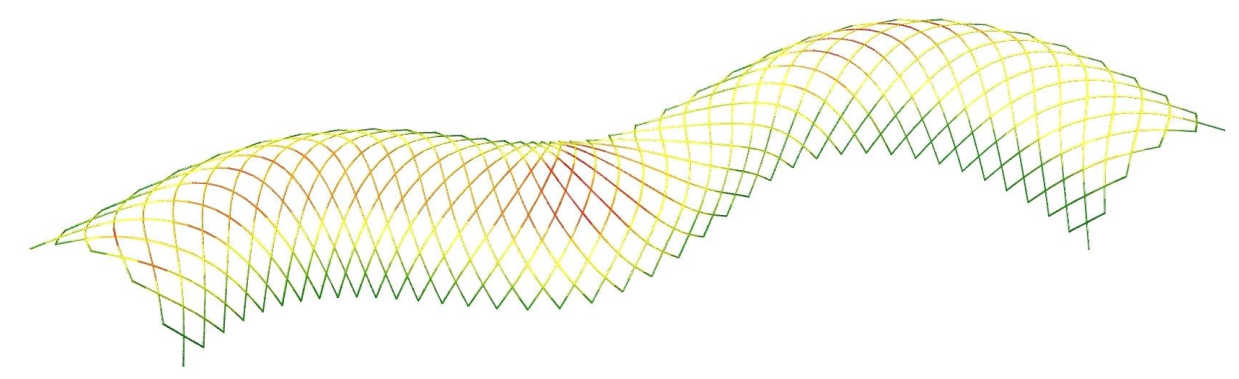

Figure 31: Smooth bending moments in the Tchebycheff net after relaxation of the length constraint in the grid of Figure 28 (high values in red and low in green).

\subsection{Form-finding with priority to the grid regularity}

The third strategy is inspired by the construction process of smaller scale realisations by the authors $[26,27,28]$ but similar to the largest gridshell ever built in Mannheim [31]. The grid here has been assembled on the ground and then lifted by cranes, controlling the form by the sole positions of the members' ends. In those cases, the final form is obtained by the static equilibrium of the grid under self-stresses induced by bending. The bracing only stabilises the form. This process is practically less demanding on the field, but allows less control of the form which is the results of mechanical constraints. 
Supposing hence that the geometry of the flat two-way grid is known and elastically brought in the configuration obtained by duality of the isoradial mesh, supposing also that the ends of each member are fixed to the ground, an equilibrium position can be found by relaxing this configuration using dynamic relaxation or any other non-linear method [32, 33]. Considering then that small variations of the prestress in the initial configuration can be obtained by increasing the ratio of the rest length of the members over the length in the initial configuration which is equal to the mesh radius $\left(l_{\text {rest }} / R\right)$, an optimisation can be conducted on this ratio in order to minimise the warping of the cover panels in the relaxed configuration. The relative length increase $\Delta l_{\text {grid }}$ is here directly given by:

$$
\Delta l_{\text {grid }}=\frac{l_{\text {rest }}-R}{R}
$$

Admissible warping for glass cover are given by [34] with a limit of $1 / 175^{\text {th }}$ of the diagonal length. One can see on Figure 32 that the number of warped panels is reduced (8 among 200), so that this construction process seems also very appropriate for the construction of elastic gridshell with planar panels.

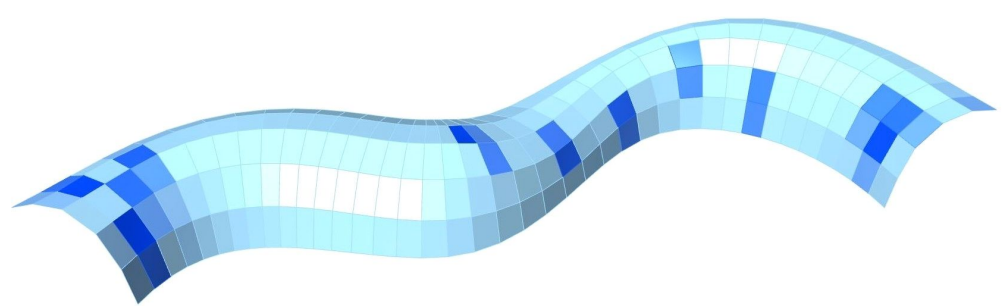

Figure 32: Warping of the panels after relaxation of the planarity constraint in the grid of Figure 28; only the 8 panels in dark blue exceed the warping criterion. 


\subsection{Comparison of the offsetting strategies}

The comparison of these three offsetting strategies is based on three surfaces: an anticlastic surface and a synclastic surface with circular directrices and generatrices (shown in Figure 33) and the open surface with alternate positive and negative Gaussian curvature shown in previous section. For the three surfaces, we analyse how the different strategies converge and the optimal offset values. The main characteristics of each strategie are recalled in Table 2.

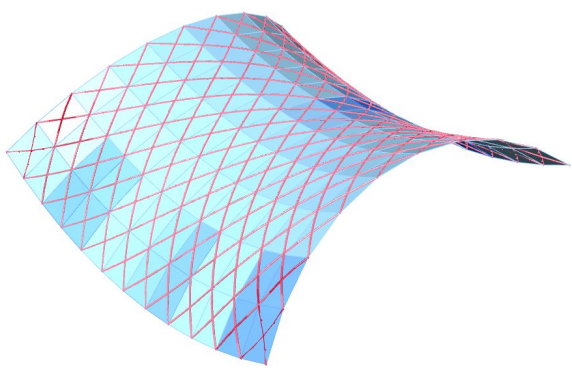

(a) Anticlastic surface

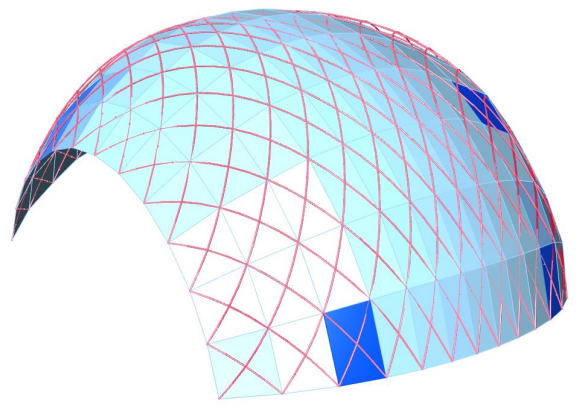

(b) Synclastic surface

Figure 33: Double curved surfaces used for offset optimisation.

Starting with the first strategy, it can be seen on Figure 34 that an optimal offset value exists for all surfaces and that this value relatively to the radius of the mesh (i.e. the ratio $\mathbf{O O}^{\prime}$ over $\mathbf{O A}$ in Figure 29) varies from one surface to another (from $3 \%$ to $6 \%$ ). The decrease of the total energy varies then from $12 \%$ until $40 \%$. The benefit is thus limited but, as the optimisation is carried out on a single value (with Newton's method for example), it is done in almost real time for the three meshes studied here which have 100 and 200 faces respectively. 


\begin{tabular}{|c|c|c|c|}
\hline Strategy & Parameters & Characteristics & Comp. time \\
\hline Geometric & Offset size & $\begin{array}{l}\text { •Regular grid } \\
\text { •Planar panels } \\
\text { • Not in mechanical equilibrium }\end{array}$ & $\begin{array}{c}\text { Low } \\
(250 \mathrm{~ms})\end{array}$ \\
\hline $\begin{array}{l}\text { DR Planar } \\
\text { panels }\end{array}$ & $E S$ & $\begin{array}{l}\text {-Quasi-regular grid } \\
\text { •Planar panels } \\
\text { •Constrained mech. equilibrium }\end{array}$ & $\begin{array}{c}\text { Low } \\
(1.2 \mathrm{~s})\end{array}$ \\
\hline $\begin{array}{l}\text { DR Regular } \\
\text { grid }\end{array}$ & $l_{\text {rest }} / R$ & $\begin{array}{l}\text {-Regular grid } \\
\text {-Quasi-planar panels } \\
\text {-True mech. equilibrium }\end{array}$ & $\begin{array}{l}\text { Medium } \\
(25 \mathrm{~s})\end{array}$ \\
\hline
\end{tabular}

Table 2: Optimal offsets for the three strategies.

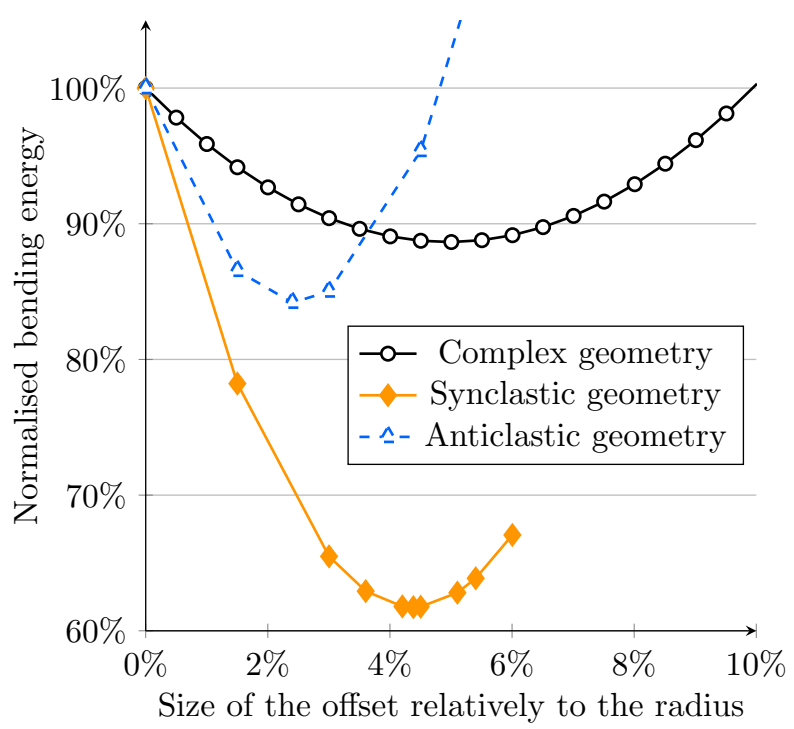

Figure 34: Influence of the relative size of the offset on the bending energy using the purely geometric strategy. 
For the second strategy, the form-finding parameters must be tuned, so that the bending forces in the members can modify their lengths. To this end,

635

plateau is limited as its maximum value is below $1 \%$ of the radius. This value is generally obtained for $E S=1.10^{-7}$. Note that the average length variation for the plateau is approximately half of the maximum length variation for the three surfaces.

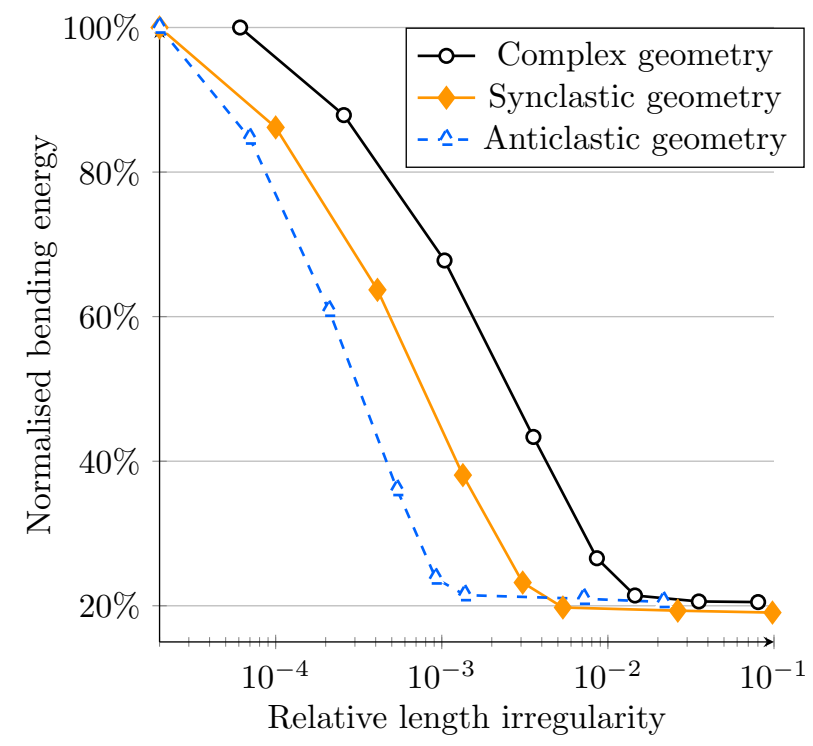

Figure 35: Influence of the grid irregularity on the bending energy with the second strategy. 
is the ratio of the rest length over the initial length and the optimisation criteria is the number of warped panels. Based on [34] which recommends that the gap remains below $l / 175^{\text {th }}$, it is found that in general, the equilibrium configuration is close to the initial configuration, so that the criteria is fulfilled for almost all panels. Actually, for any value of the ratio $\frac{l_{\text {rest }}-l_{\text {init }}}{l_{\text {init }}}$ below $1 \%$, the criteria is always fulfilled for the two simple structures, and for $98 \%$ of the panels of the complex shape.

To analyse more precisely the influence of $l_{\text {rest }} / l_{\text {init }}$, two criteria have been studied: the number of panels with a warping exceeding $1 / 500^{\text {th }}$ and the normalised warping energy of the panels. These two criteria being strongly correlated in practice, the results will be presented in terms of normalised warping energy only (see Figure 36). It is observed that the variations of the warping energy with $l_{\text {rest }} / l_{\text {init }}$ are not monotonous but that its minimum approximately coincides with the ratio that would give an equivalent offset value similar to the one minimising the bending energy in the first strategy.

Besides, it is observed that the absolute values of the bending energy in the two form-finding processes are equivalent. It can thus be concluded that, from a mechanical point of view, both relaxation strategies are equally valuable and lead similar elastic prestresses in the deformed grid.

It is also worth remarking that, after the form-finding under planarity constraint, the average length extension under regularity constraint $\Delta l_{\text {grid }}$ is similar to the optimal value of the geometrical optimisation $\Delta l_{\text {geom }}$, which is slightly lower than the optimal value obtained after relaxation under planarity constraint $\Delta l_{\text {planar }}$. For this reason, we suggest that in a first step the optimal value of the geometrical offset is determined by a purely real- 
time geometrical process and then that this value is used to relax the grid in the neighbourhood of the isoradial mesh which will result in a structure at equilibrium with a very limited warping of the panels.

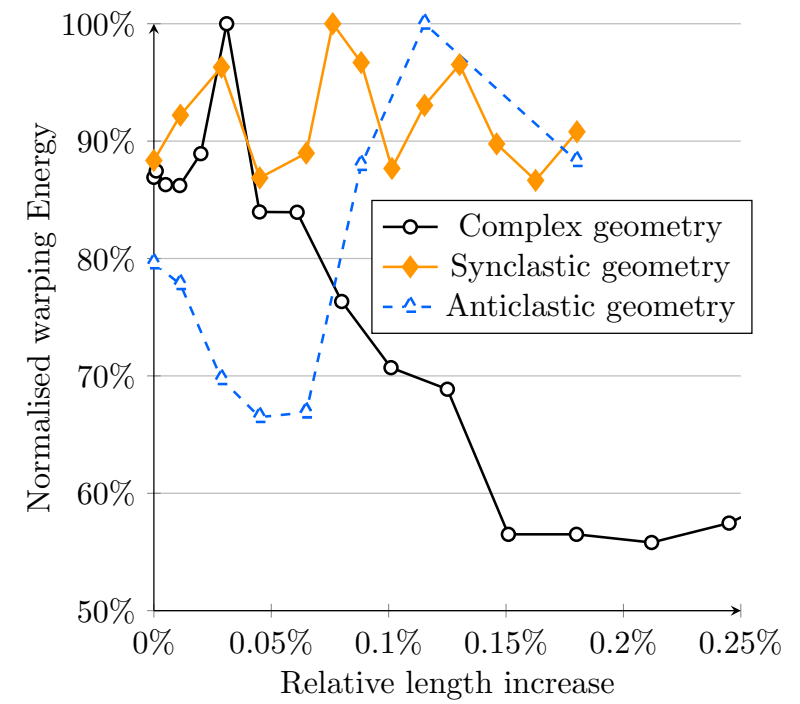

Figure 36: Influence of the relative length increase on the warping energy.

\begin{tabular}{|c|c|c|c|c|}
\hline Strategy & Criterion & Complex & Anticlastic & Synclastic \\
\hline Geometric & $\Delta l_{\text {geom }}$ & $1.8 \%$ & $0.3 \% 0$ & $1.0 \% 0$ \\
Planarity & $\Delta l_{\text {planar }}$ & $3.9 \%$ & $0.6 \% 0$ & $2.4 \% 0$ \\
Regularity & $\Delta l_{\text {grid }}$ & $2.1 \%$ & $0.4 \% 0$ & $1.2 \% 0$ \\
\hline
\end{tabular}

Table 3: Optimal offsets for the three strategies.

\section{Conclusion}

A new bottom-up approach in the design of free-form structures is proposed in this paper. It allows the construction of Tchebycheff nets from 
particular circular meshes which are built with unique radii. A practical consequence is that it is then possible to build elastic gridshells which can be covered with planar facets and whose bracing is naturally optimised in method presented in this article. It would probably give more insight on some difficulties encountered here, like the problem of closed isoradial meshes for any curve. 


\section{References}

[1] M. Bagneris, R. Motro, B. Maurin, N. Pauli, Structural morphology issues in conceptual design of double curved systems, International Journal of Space Structures 23 (2) (2008) 79-87.

[2] F. Otto, IL10 Gitterschalen, Institut für leichte Flächentragwerke (IL), Stuttgart, 1974.

[3] C. Douthe, O. Baverel, J.-F. Caron, Form-finding of a grid shell in composite materials, Journal of the I.A.S.S. 47 (150) (2006) 53-62.

[4] E. Lafuente-Hernandez, O. Baverel, C. Gengnagel, On the design and construction of elastic gridshells with irregular meshes, International Journal of Space Structures 28 (3-4) (2013) 161-174. doi:10.1260/ $0266-3511.28 .3-4.161$.

[5] E. Lafuente-Hernandez, C. Gengnagel, S. Sechelmann, T. Rörig, On the Materiality and Structural Behaviour of Highly-Elastic Gridshell Structures, in: A. Gengnagel, C.AND Kilian, N. Palz, F. Scheurer (Eds.), Computing Design Modelling Symposium, 2011, pp. 123-135.

[6] B. Lefèvre, C. Douthe, O. Baverel, Buckling of elastic gridshells, Journal of the International Association for Shell and Spatial Structures 56 (3) (2015) 153-171.

[7] E. Ghys, Sur la coupe des vêtements. variation autour d'un thème de tchebychev, Enseign. Math. 57 (1/2) (2011) 165-208. doi:10.4171/ LEM/57-1-8. 
[8] Y. Masson, L. Monasse, Existence of global chebyshev nets on surfaces of absolute gaussian curvature less than $2 \pi$, Journal of Geometry (2016) 1-8doi:10.1007/s00022-016-0319-1.

725

[14] J. Schlaich, H. Schober, Glass roof for the hippo zoo at berlin, Structural Engineering International 7: (4) (1997) 252-254.

[15] J. Glymph, D. Shelden, C. Ceccato, J. Mussel, H. Schober, A parametric strategy for free-form glass structures using quadrilateral planar facets, 
Automation in Construction 13 (2) (2004) 187-202. doi:10.1016/j. autcon.2003.09.008.

[16] R. Mesnil, C. Douthe, O. Baverel, B. Léger, J.-F. Caron, Isogonal moulding surfaces: A family of shapes for high node congruence in free-form structures, Automation in Construction 59 (2015) 38-47. doi:10.1016/j.autcon.2015.07.009.

[17] R. Mesnil, Structural explorations of fabrication-aware design spaces for non-standard architecture, Ph.D. thesis, Universit Paris-Est (2017).

[18] Y. Liu, W. Wang, H. Pottmann, J. Wallner, Y. Yong-Liang, Geometric modeling with conical meshes and developable surfaces, ACM Transaction on Graphics 25 (3) (2006) 681-689. doi:10.1145/1141911. 1141941.

[19] C. Boutillier, B. de Tilière, Probability in Complex Physical Systems, Springer, 2011, Ch. Statistical Mechanics on Isoradial Graphs, pp. 491512.

[20] Y. Liu, W. Wang, On Vertex Offsets of Polyhedral Surfaces, in: H. Pottman, A. Kilian, M. Hofer (Eds.), Advances in Architectural Geometry, 2008, pp. 61-64.

[21] R. Mesnil, C. Douthe, O. Baverel, B. Léger, Marionette mesh: from descriptive geometry to fabrication-aware design, in: Advances in Architectural Geometry, 2016. doi:10.3218/3778-4.

[22] O. Kelly, R. Harris, M. Dickson, The Construction of the Downland Gridshell, The Structural Engineer 79 (17) (2001) 25-33. 
[23] D. Naicu, R. Harris, C. Williams, Timber gridshells: Design methods and their application to a temporary pavilion., in: S. A. (Ed.), World Conference on Timber Engineering (WCTE), Quebec City, 2014.

[24] R. Harris, S. Haskins, J. Roynon, The Savill Garden gridshell: Design and construction, The Structural Engineer 86 (17) (2008) 27-34.

[25] K. Bollinger, M. Grohmann, K. Loffler, W. A., Planungsprozess komplexer struckturen: Interaktion zwischen architektur und tragwerk, Bautechnik 91 (4). doi:10.1002/bate.201400023.

[26] C. Douthe, J.-F. Caron, O. Baverel, Gridshell structures in glass fibre reinforced polymers, Construction and Building Materials 24 (9) (2010) 1580-1589. doi:10.1016/j . conbuildmat.2010.02.037.

[27] F. Tayeb, J.-F. Caron, O. Baverel, L. Du Peloux, Stability and robustness of a $300 \mathrm{~m} 2$ composite gridshell structure, Construction and Building Materials 49 (2013) 926-938. doi:10.1016/j.conbuildmat. 2013. 04.036 .

[28] L. Du Peloux, F. Tayeb, O. Baverel, J.-F. Caron, Construction of a large composite gridshell structure, Structural Engineering International 26 (2) (2016) 160-167. doi:10.2749/101686616X14555428758885.

[29] M. McQuaid, Shigeru Ban, Phaidon, 2006.

[30] C. Douthe, O. Baverel, Morphological and mechanical investigation of double-layer reciprocal structures, Nexus Network Journal 16 (1) (2014) 191-206. doi:10.1007/s00004-014-0185-9. 
790

[31] E. Happold, W. Liddell, Timber lattice roof for the Mannheim Bundesgartenschau, The Structural Engineer 53 (3) (1975) 99-135.

[32] C. Douthe, O. Baverel, Design of reciprocal frames with the dynamic relaxation method, Computers And Structures 87 (21) (2009) 12961307. doi:10.1016/j.compstruc.2009.06.011.

[33] R. Mesnil, C. Douthe, J. Ochsendorf, Stability of pseudo-funicular elastic grid shells, International Journal of Space Structures 30 (1) (2015) 2736. doi:10.1260/0266-3511.30.1.27.

[34] W. Laufs, G. Vikner, Gekrümmte glasflächen - ein zusammenspiel von geometrie und glasdetaillierung., Stahlbau Spezial Konstruktiver Glasbau (2010) 16-21. doi:10.1002/stab. 201001312. 